\title{
Discovery of Water at High Spectral Resolution in the Atmosphere of 51 Peg b
}

\author{
J. L. Birkby ${ }^{1,2,5}$, R. J. de Kok ${ }^{2,3}$, M. Brogi ${ }^{4,6}$, H. Schwarz ${ }^{2}$, and I. A. G. Snellen ${ }^{2}$ \\ ${ }^{1}$ Harvard-Smithsonian Center for Astrophysics, 60 Garden Street, Cambridge, MA 02138, USA; jbirkby@cfa.harvard.edu \\ ${ }^{2}$ Leiden Observatory, Leiden University, Niels Bohrweg 2, 2333 CA Leiden, The Netherlands \\ ${ }^{3}$ SRON Netherlands Institute for Space Research, Sorbonnelaan 2, 3584 CA Utrecht, The Netherlands \\ ${ }^{4}$ Center for Astrophysics and Space Astronomy, University of Colorado at Boulder, Boulder, CO 80309, USA \\ Received 2016 June 13; revised 2017 January 12; accepted 2017 January 24; published 2017 March 2
}

\begin{abstract}
We report the detection of water absorption features in the day side spectrum of the first-known hot Jupiter, 51 Peg $\mathrm{b}$, confirming the star-planet system to be a double-lined spectroscopic binary. We use high-resolution $(R \approx$ 100,000), $3.2 \mu \mathrm{m}$ spectra taken with CRIRES/VLT to trace the radial-velocity shift of the water features in the planet's day side atmosphere during $4 \mathrm{hr}$ of its 4.23 day orbit after superior conjunction. We detect the signature of molecular absorption by water at a significance of $5.6 \sigma$ at a systemic velocity of $V_{\text {sys }}=-33 \pm 2 \mathrm{~km} \mathrm{~s}^{-1}$, coincident with the $51 \mathrm{Peg}$ host star, with a corresponding orbital velocity $K_{\mathrm{P}}=133_{-3.5}^{+4.3} \mathrm{~km} \mathrm{~s}^{-1}$. This translates directly to a planet mass of $M_{\mathrm{p}}=0.476_{-0.031}^{+0.032} M_{\mathrm{J}}$, placing it at the transition boundary between Jovian and Neptunian worlds. We determine upper and lower limits on the orbital inclination of the system of $70^{\circ}<i<82^{\circ} .2$. We also provide an updated orbital solution for $51 \mathrm{Peg} \mathrm{b}$, using an extensive set of 639 stellar radial velocities measured between 1994 and 2013, finding no significant evidence of an eccentric orbit. We find no evidence of significant absorption or emission from other major carbon-bearing molecules of the planet, including methane and carbon dioxide. The atmosphere is non-inverted in the temperature-pressure region probed by these observations. The deepest absorption lines reach an observed relative contrast of $0.9 \times 10^{-3}$ with respect to the host star continuum flux at an angular separation of 3 milliarcseconds. This work is consistent with a previous tentative report of K-band molecular absorption for 51 Peg b by Brogi et al.
\end{abstract}

Key words: planetary systems - planets and satellites: composition - planets and satellites: gaseous planets planets and satellites: individual (51 Peg b)

Supporting material: machine-readable table

\section{Introduction}

The field of exoplanets has come of age, with twenty-one years passing since the first confirmation of an exoplanet orbiting a main sequence star, 51 Peg b (Mayor \& Queloz 1995). The close, 4.23 day orbit of this planet placed it in an entirely new and unexpected population of highly irradiated bodies close to their parent stars. It ignited the field of planet migration theory (Lin et al. 1996; Rasio \& Ford 1996), and paved the way for another 3434 confirmed exoplanets in 2568 planetary systems to date. ${ }^{7}$ In just two decades, exoplanets have transitioned from mere theoretical possibility to highly characterizable systems. There are now radius measurements of Earth-like planets, aided by asteroseismology, with error bars precise to $120 \mathrm{~km}$ (Ballard et al. 2014); there is evidence that clouds pervade the atmospheres of exoplanets across the mass spectrum from super-Earths to hot Jupiters (e.g., Evans et al. 2013; Kreidberg et al. 2014; Heng 2016; Sing et al. 2016); there are a growing number of robust detections of elemental and molecular species in transiting planets using the Hubble Space Telescope, including sodium, potassium, and water (see e.g., Crossfield 2015 for an up-to-date review of chemicals observed in exoplanet atmospheres), alongside the first detections of carbon monoxide, water, and methane in the atmospheres of widely separated directly imaged giants planets (e.g., Konopacky et al. 2013; Snellen et al. 2014; Barman

\footnotetext{
5 NASA Sagan Fellow.

6 NASA Hubble Fellow.

7 As of 2016 June 13, see http://www.exoplanet.eu, Schneider et al. (2011).
}

et al. 2015; Macintosh et al. 2015), and in the atmospheres of non-transiting hot Jupiters using ground-based high-resolution spectroscopy (Brogi et al. 2012; Rodler et al. 2012; Lockwood et al. 2014). Even the global wind dynamics and atmospheric circulation of hot Jupiters have been studied in detail (e.g., Knutson et al. 2009; Stevenson et al. 2014; Louden \& Wheatley 2015; Brogi et al. 2016a; Zhou et al. 2016). The next few decades hold promise of remote, ground-based biomarker hunting in Earth-like planets orbiting nearby bright stars (e.g., Snellen et al. 2013; Rodler \& López-Morales 2014), as well as the mapping of features akin to Jupiter's Great Red Spot in the atmospheres of giant exoplanets with the extremely large telescopes (Kostov \& Apai 2013; Crossfield 2014; Snellen et al. 2014; Karalidi et al. 2015), in a similar vein to that already achieved for brown dwarfs (Crossfield et al. 2014). The discovery of 51 Peg b was, in short, transformational.

However, its discovery was initally met with uncertainty and caution, given its unusual orbital parameters. It was suggested that the radial velocity measurements that revealed the planet were instead line profile variations caused by non-radial stellar oscillations (Gray 1997; Gray \& Hatzes 1997). Although this claim was later retracted in light of additional observations (Gray 1998), the rapid onslaught of similar discoveries (e.g., Butler et al. 1997), and the eventual detection of transiting exoplanets (Charbonneau et al. 2000; Henry et al. 2000), largely laid to rest any doubts about the planetary nature of the non-transiting planet orbiting $51 \mathrm{Peg}$. As a final proof, in this paper, we demonstrate the true binary nature of the 51 Peg starplanet system, revealing it to be a double-lined spectroscopic (non-eclipsing) binary, via the direct detection of water 
absorption lines in the spectrum of the planet's atmosphere that undergo a change in Doppler-shift.

The technique employed in this work uses ground-based, high-resolution spectroscopy to directly observe the large radial velocity change $\left(\Delta \mathrm{RV}_{\mathrm{P}} \sim \mathrm{km} \mathrm{s}^{-1}\right)$ of the planet's spectrum while the contamination from Earth's telluric features and the stellar lines are essentially stationary $\left(\Delta \mathrm{RV}_{\star} \sim \mathrm{m} \mathrm{s}^{-1}\right)$. It works on the premise that at high resolution (e.g., $R \sim 100,000$ ), broad molecular bands are resolved into a dense forest of tens to hundreds of individual lines in a pattern that is unique to each molecule. Consequently, a significant correlation between a highresolution molecular template and the observed planetary spectrum, at a systemic velocity that is coincident with the host star, is evidence of the presence of a specific molecule in the planet's atmosphere that is difficult to mimic with instrumental or Earth-atmosphere systematics. The concept of using high-resolution spectroscopy in this manner to study exoplanet atmospheres arose not long after the discovery of 51 Peg b. Charbonneau et al. (1998) initially considered that reflected light from 51 Peg b may have been responsible for the line profile variations proposed by Gray \& Hatzes (1997), which led to searches with high-resolution optical spectroscopy to directly detect hot Jupiters via their reflected light. Charbonneau et al. (1999) and Collier Cameron et al. (1999) announced upper limits and even a detection, respectively, of the reflected light from $\tau$ Boo b, although ultimately they converged to an upper limit on the star-planet flux ratio of $F_{\mathrm{p}} / F_{\mathrm{s}}<3.5 \times 10^{-5}$ (Collier-Cameron et al. 2004). Many of the diagnostic properties of high-resolution spectra of exoplanets were outlined by Brown (2001) and multiple attempts followed to directly detect the thermally emitted light from giant exoplanets at infrared wavelengths using highresolution spectrographs such as NIRSPEC on Keck II and Phoenix on Gemini South (e.g., Brown et al. 2002; Deming et al. 2005; Barnes et al. 2007a, 2007b), but again only providing upper limits. It wasn't until Snellen et al. (2010) used the CRyogenic high-resolution InfraRed Echelle Spectrograph (CRIRES) at the Very Large Telescope (VLT) that the technique delivered its first unambiguous detections of a molecule (carbon monoxide) in the atmosphere of an exoplanet. While weather may have thwarted some earlier attempts with other telescopes, the stability of CRIRES, in part delivered by its use of adaptive optics and Nasmyth mounting, and its higher spectral resolution, were undoubtedly instrumental to its success. Since then, the technique has been used to study the atmospheric composition of both transiting (Crossfield et al. 2011; Birkby et al. 2013; de Kok et al. 2013; Rodler et al. 2013; Hoeijmakers et al. 2015; Schwarz et al. 2015; Brogi et al. 2016a) and non-transiting exoplanets (Brogi et al. 2012; Rodler et al. 2012; Lockwood et al. 2014; Snellen et al. 2014; Piskorz et al. 2016; Schwarz et al. 2016). Additionally, the technique reveals the inclination, $i$, of the orbit, thus the mass of the planet can be measured directly in both cases, rather than just a lower $M_{\mathrm{P}} \sin (i)$ limit for the non-transiting planets.

The use of high-resolution infrared ground-based spectroscopy in this paper further cements the important role of high-resolution optical and infrared spectrographs in studying the atmospheres of non-transiting planets. This is pertinent given that the nearest potentially habitable, non-transiting, terrestrial planets orbiting small stars (which have the most favorable contrast ratios) are likely to be a factor of four times closer to Earth than their transiting counterparts (Dressing \& Charbonneau 2015). Finally, this paper also serves as an independent confirmation of the tentative detection of molecular absorption features in $51 \mathrm{Peg} \mathrm{b}$ reported in Brogi et al. (2013). While we have used the same instrument as Brogi et al. (2013) for our observations, we operated under a different instrument setup to observe a redder wavelength region, and our method for removing telluric contamination also differs; thus we consider our results to be independent.

The paper is presented as follows: Section 2 describes our observations, the instrumental set-up, and the reduction of the resulting spectra of $51 \mathrm{Peg} \mathrm{b}$, including the process of removing telluric contamination. In Section 3, we detail the crosscorrelation process used to extract the planetary signal, and present our results. This includes an update to the orbital solution and ephemeris for 51 Peg b. Section 4 presents a discussion of our findings and compares them with preliminary reports of molecular absorption in the atmosphere of $51 \mathrm{Peg} b$ by Brogi et al. (2013). We conclude in Section 5.

\section{Observations and Data Reduction}

\subsection{Observations}

We observed the bright star 51 Peg $(\mathrm{G} 2.5 \mathrm{~V}, V=5.46 \mathrm{mag}$, $K=3.91 \mathrm{mag}$ ) for $3.7 \mathrm{hr}$ during the night beginning 2010 October 21, using CRIRES $^{8}$ (Kaeufl et al. 2004) mounted at Nasmyth A at the VLT (8.2 m UT1/Antu), Cerro Paranal, Chile. The observations were collected as part of the ESO large program 186.C-0289. The instrument setup consisted of a 0.2 arcsec slit centred on $3236 \mathrm{~nm}$ (order 17), in combination with the Multi-Application Curvature Adaptive Optic system (MACAO; Arsenault et al. 2003). The CRIRES infrared detector is comprised of four Aladdin III InSb-arrays, each with $1024 \times 512$ pixels, and separated by a gap of 280 pixels. The resulting wavelength coverage of the observations was $3.1806<\lambda(\mu \mathrm{m})<3.2659$ with a resolution of $R \approx 100,000$ per resolution element (see Figure 1).

We observed 51 Peg continuously while its hot Jupiter companion passed through orbital phases $0.55 \lesssim \phi \lesssim 0.58$, corresponding to an expected change in the planet's radial velocity of $\triangle \mathrm{RV}_{\mathrm{P}}=-23 \mathrm{~km} \mathrm{~s}^{-1}$ (15 pixels on the CRIRES detectors). In total, we obtained 42 spectra, with the first 20 spectra each consisting of two sets of $5 \times 20 \mathrm{~s}$ exposures, and the remainder each consisting of two sets of $5 \times 30 \mathrm{~s}$ exposures. The increase in the exposure time was aimed at maintaining a constant signal-to-noise ratio $(\mathrm{S} / \mathrm{N})$ in the continuum of the observed stellar spectra after a sudden and significant deterioration of the seeing (increasing from 0.75 to 1.4 arcsec between one set of frames, see Section 2.3). To enable accurate sky-background subtraction, the telescope was nodded along the slit by 10 arcsec between each set of exposures in a classic ABBA sequence, with each of the final 42 extracted spectra consisting of an $\mathrm{AB}$ or BA pair. A standard set of CRIRES calibration frames was taken the following morning. Later in this paper, we will compare our results to those of Brogi et al. (2013), who observed 51 Peg b at $2.3 \mu \mathrm{m}$ at the same spectral resolution with CRIRES/VLT on

\footnotetext{
8 CRIRES was dismounted from UT1/VLT in the summer of 2014 to be upgraded to CRIRES+, which will have improved detectors and a wider wavelength coverage (Follert et al. 2014). Its return is eagerly anticipated.
} 


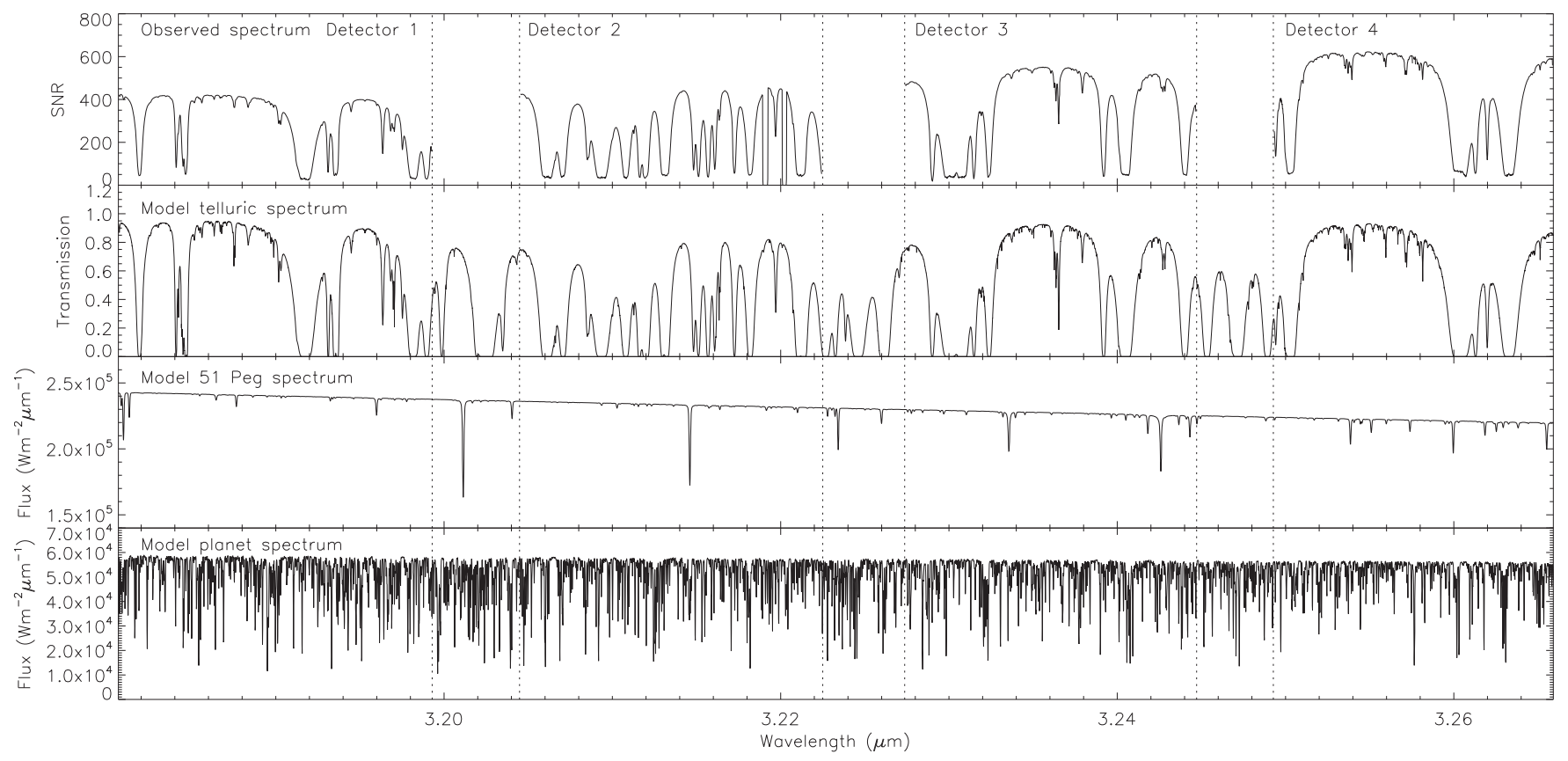

Figure 1. Top: the photon-limited average signal-to-noise of the 51 Peg spectra observed with CRIRES/VLT. The vertical dotted lines mark the boundaries of the gaps between the detectors. Second panel: a model telluric transmission spectrum from ATRAN assuming a precipitable water vapour PWV $=2 \mathrm{~mm}$ at Cerro Paranal. The observed spectra are completely dominated by the tellurics. Third panel: for visual purposes only, an approximate stellar model for 51 Peg, assumed here to be the solar spectrum, shifted to match the velocity of $51 \mathrm{Peg}$ during our observations. The spectrum was obtained at $R=100,000$. Most of the strong stellar lines fall between the detector gaps. Bottom panel: an example of one of our water molecular template spectra for 51 Peg b (see Section 3.1), shifted to the velocity of 51 Peg during our observations. Note the many tens of strong absorption lines.

dates either side of these observations, including 2010 October 16,17 , and 25 .

\subsection{Data Reduction}

Throughout the data reduction, the four CRIRES detectors were treated independently and separately. We used the CRIRES ESOREX pipeline (v2.2.1) to first process the observed 2D images, including nonlinearity and bad pixel corrections, flat-fielding, background subtraction and combination of the nodded exposures, and finally the optimal extraction of the 1D spectra. The 42 extracted spectra were stored as four $1024 \times 42$ matrices. The matrix for detector 1 is shown in the upper panel of Figure 2. The $x$-axis corresponds to pixel number (i.e., wavelength channel) and the $y$-axis denotes the frame number (i.e., orbital phase or time). Remaining singular bad pixels, bad regions, and bad columns in these matrices were identified iteratively by eye and replaced by spline interpolation values from their horizontal neighboring pixels. There was a total of $0.4-1.0$ per cent bad pixels in each matrix, with detector 4 requiring the most corrections.

A gradual drift occurs in the position of the spectrum in the dispersion direction on the detectors over the course of the observations. To correct this, we apply a global shift to each spectrum on each detector using a spline interpolation to align it to the telluric features of the spectrum with the highest $\mathrm{S} / \mathrm{N}$. The shifts were determined by cross-correlating the spectrum in question with the highest $\mathrm{S} / \mathrm{N}$ spectrum using IRAF.FXCOR. Detector 1 required the largest corrections, with the last spectrum deviating from the first by 0.7 pixels (the equivalent of $1 \mathrm{~km} \mathrm{~s}^{-1}$ or $0.01 \AA$ ). Brogi et al. (2013) note that their $2.3 \mu \mathrm{m}$ observations of $51 \mathrm{Peg} \mathrm{b}$ also experience a similar drift, which correlates with the temperatures of the instrument pre-optics system, grating, and its stabilizer. For small fluctuations $(<0.05 \mathrm{~K})$, the drift did not exceed 0.5 pixels, but a $1.5 \mathrm{~K}$ change in these temperatures resulted in much larger drifts (1.5 pixels) for their observations on 2010 October 25 , which resulted in a non-detection of the $51 \mathrm{Peg} \mathrm{b}$ signal. The drift in our $3.5 \mu \mathrm{m}$ observations does not correlate with these instrumental temperatures, which remained stable throughout the night. Instead, the drift correlates with the ambient temperature of the telescope dome and the primary mirror temperature, which both cooled by $2 \mathrm{~K}$ over the course of the observations. However, the drift of our $3.5 \mu \mathrm{m}$ spectra is comparatively small, thus we do not expect the alignment correction to significantly affect our subsequent analysis.

Finally, a common wavelength solution per detector was calculated using a synthetic telluric transmission spectrum (see the second panel of Figure 1) from ATRAN ${ }^{9}$ (Lord 1992) to identify the wavelengths of the telluric features in the highest $\mathrm{S} / \mathrm{N}$ spectrum. Line positions were identified using IRAF. IDENTIFY, and fitted with a third-order Chebyshev polynomial to obtain the wavelength solution. This replaced the default solution from the CRIRES pipeline from which it differed by up to $1.9 \AA$. The wavelength solutions were not linearized and thus retained the pixel-spacing information. Detector 2 contains significant telluric contamination (see Figures 1 and 2) such that no useful planetary signal can be extracted. Following Birkby et al. (2013), we therefore discarded detector 2 and exclude it from all further analyses in this paper.

\footnotetext{
9 http://atran.sofia.usra.edu/cgi-bin/atran/atran.cgi
} 


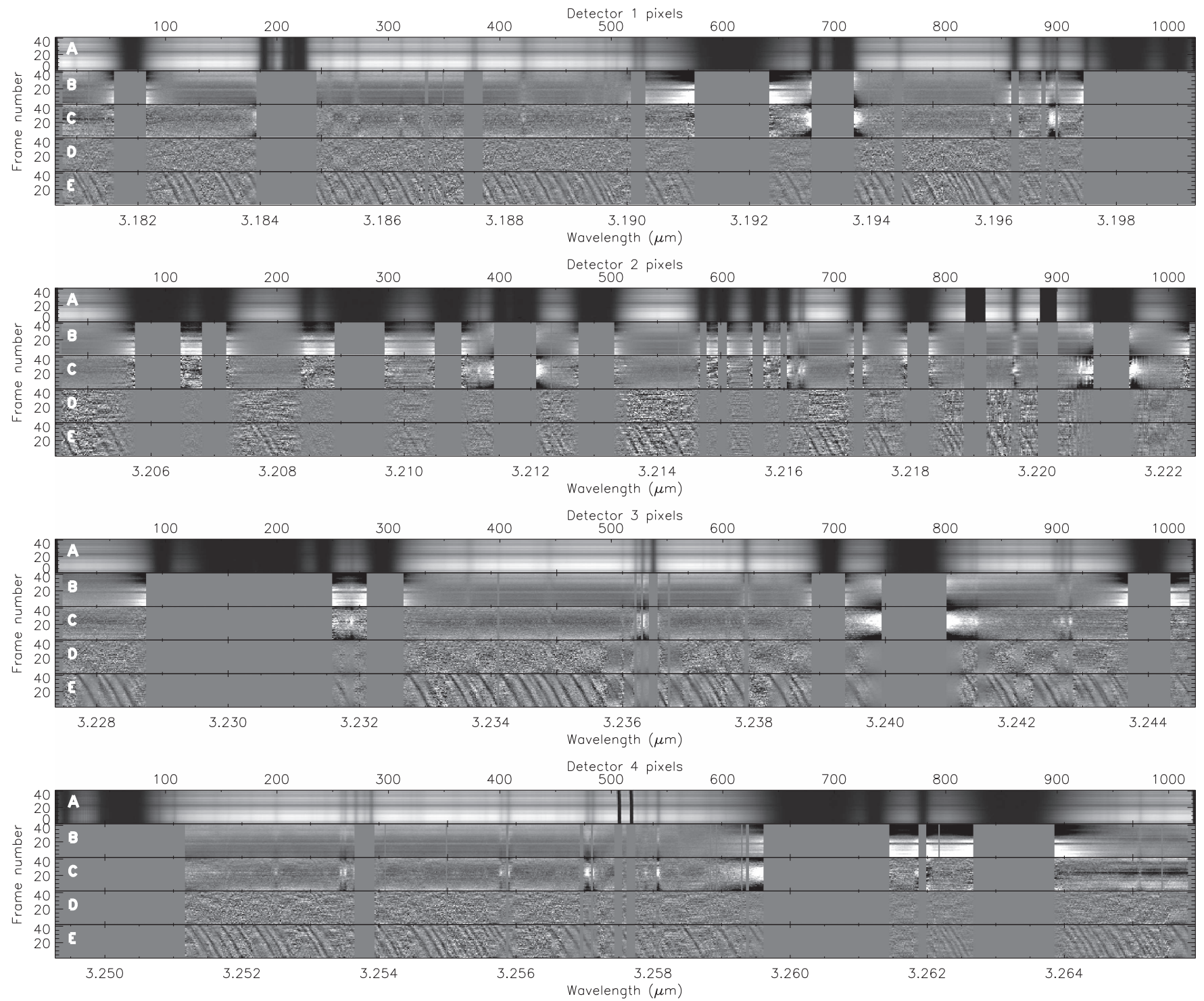

Figure 2. Spectra at different stages of the telluric removal process for each CRIRES detector. The $x$-axes correspond to wavelength i.e., pixel number, and the $y$-axes are ordered in time i.e., frame number. Detector 2 is not used in our analysis but it shown here for completeness. The sub-panels are as follows. Panel (A): the spectra extracted from the CRIRES pipeline, with bad pixels corrected, and aligned to match the telluric features of the highest $\mathrm{S} / \mathrm{N}$ spectrum. The dark horizontal bands contain spectra taken under poor seeing. The broad dark vertical bands are saturated telluric lines. Panel (B): as in A, but normalized and with the mean of each column subtracted from itself. The solid gray regions mark regions of saturated telluric features which are excluded from our cross-correlation analysis (see Section 3.2). Panel (C): the residuals remaining after one iteration of SYSREM on the spectra. Note that the non-saturated telluric and stellar features e.g., at pixel 420 on detector 1 still remain. Panel (D): the residuals after applying the adopted number of iterations of SYSREM for the detector, the high-pass filter, and dividing each column by its variance. The telluric features have been sufficiently removed, leaving behind the planet spectrum buried in the noise. Panel (E): the same as (D), but with a bestmatching model planet spectrum from Section 3.2 injected at the expected Doppler shift of $51 \mathrm{Peg} \mathrm{b}$ at a factor of 100 times greater than its nominal value before running SYSREM. This is to highlight the many individual strong water lines in the planet spectrum whose signal will be combined with the cross-correlation procedure detailed in Section 3.2. The authors are happy to supply the processed spectral matrices upon request.

\subsection{Removal of Telluric Contamination}

Telluric absorption from the Earth's atmosphere is the dominant spectral feature in our observed spectra (see Figure 1), while the Doppler-shifted features of 51 Peg b are expected at the $10^{-3}-10^{-4}$ level with respect to the stellar continuum. Thus, we needed to remove the telluric contamination. In a previous analysis of high-resolution spectra of 51 Peg b, Brogi et al. (2013) included an additional step to remove stellar lines before removing the telluric features. However, they studied the $2.3 \mu \mathrm{m}$ region, which contains multiple strong $\mathrm{CO}$ lines from the Sun-like host star. In the $3.2 \mu \mathrm{m}$ region under consideration here, a comparison with a proxy solar model spectrum for 51
Peg from Robert Kurucz's stellar model database ${ }^{10}$ at $R=$ 100,000 indicates that there are few strong absorption lines from the host star, and that they mostly fall on gaps between detectors, or on the discarded detector 2 (see the third panel of Figure 1). Consequently, we do not perform any pre-removal of the stellar lines in the $3.2 \mu \mathrm{m}$ data set.

The removal of the telluric features in our spectra was achieved using our implementation of SYSREM, which is an algorithm based on principle component analysis but also allows for unequal error bars per data point (Tamuz et al. 2005;

\footnotetext{
${ }^{10}$ http://kurucz.harvard.edu/stars/Sun/
} 


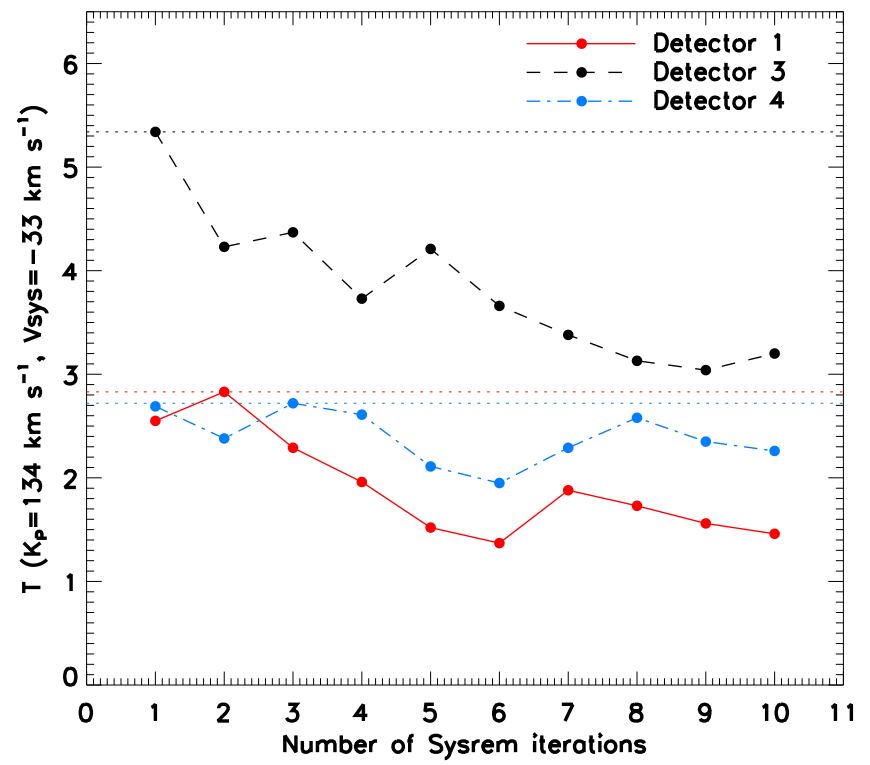

Figure 3. Detection strength ( $T$, as described in Section 3.3) of an injected fake planet at the proposed planet velocity parameters from Brogi et al. (2013) for each CRIRES detector after each iteration of SYSREM. The horizontal dotted lines mark the maximum detection strength per detector for the injected model.

Mazeh et al. 2007). It is commonly used in ground-based widefield transit surveys to correct systematic effects common to all light curves (e.g., SuperWASP; Collier Cameron et al. 2006). Each wavelength channel (i.e., pixel column) in the matrix shown in panel B of Figure 2 was treated as a "light curve", where the errors per data point are the quadrature sum of the Poisson noise and the error from the optimal extraction of the spectrum at a given pixel. Each column had its mean subtracted before being passed through SYSREM, and regions of saturated telluric features, which contain essentially no flux, were also masked. These masked regions are marked by the vertical solid gray regions in Figure 2. SYSREM then searched for common modes between the 1024 light curves per matrix, such as variation with airmass, and subtracted them, resulting in the removal of the quasi-static telluric and stellar lines, leaving only the Doppler-shifting planet spectrum in each spectrum plus noise. However, in practice, once the dominant telluric and stellar spectral features are removed, SYSREM will begin to remove the planet features too. This is because the sub-pixel shift of the planet spectrum between frames creates a small but detectable common mode between adjacent columns. Thus, we must determine when to halt the SYSREM algorithm before it removes the planet signal. To do this, we injected the best-matching model planet spectrum proposed by Brogi et al. (2013) based on observations at $2.3 \mu \mathrm{m}$ at their measured planet velocity and ephemeris $\left(V_{\text {sys }}=-33 \mathrm{~km} \mathrm{~s}^{-1}, K_{\mathrm{P}}=\right.$ $134 \mathrm{~km} \mathrm{~s}^{-1}$, with a phase shift of $\left.\Delta \phi=0.0095\right)$, and iterated SYSREM ten times. The model was injected at a nominal strength of 1. At each iteration, we used the cross-correlation method described in Sections 3.2 and 3.3 to determine the significance of the detection at the injected velocity. The results of this analysis are shown in Figure 3 for each detector. We find that SYSREM begins to remove the planet signal after only one iteration on detector 3 , but that this does not occur until after two and three iterations on detectors 1 and 4, respectively. The difference in iterations required per chip may be partly due to a known "odd-even" effect, ${ }^{11}$ which only affects detectors 1 and 4 . It is caused by variations in the gain between neighboring columns along the spectral direction. This odd-even effect has been seen previously in similar analyses of high-resolution spectroscopy of exoplanets from CRIRES/VLT (Brogi et al. 2012, 2013, 2014, 2016a; Birkby et al. 2013).

Guided by the results of executing SYSREM on the injected signal as described above, we adopted SYSREM iterations of two, one, and three for detectors 1,3 , and 4, respectively, for the analysis of the observed data (see Section 3.2). The standard deviation of the final residuals in panels D of Figure 2 are $0.0050,0.0380 .0082,0.0040$, for detectors $1,2,3$, and 4, respectively. The trends removed from each detector during each SYSREM iteration are shown in Figure 4. Possible physical causes of these trends are shown in Figure 5, and these are discussed in further detail in Section 3.4.

The final two steps in the telluric removal process were: (i) the application of a high-pass filter with a 64 pixel width smoothing function, which removes a heavily smoothed version of each residual spectrum from itself to filter out low-order variation across the matrix, and then (ii) each column is divided by its variance to account for variation in $\mathrm{S} / \mathrm{N}$ as a function of wavelength. The final product of this process for detector 1 is shown in panel (D) of Figure 2. For illustrative purposes, the bottom panel of Figure 2 shows how the final matrix would appear if a model planet was injected at the planet velocity but at $100 \times$ nominal value before running SYSREM. Many individual lines from the planet spectrum are clearly visible as they blueshift across the matrix. With the tellurics and stellar continuum removed from each detector, we could proceed to extract the observed planetary signal contained within the noise of the residual spectra.

\section{Cross-correlation Analysis and Results}

The spectral features of the molecules in the planetary atmosphere are buried in the noise of the residuals after removing the telluric contamination. To identify them, the signals from all the individual spectral lines are combined by cross-correlating the residuals with high-resolution molecular spectral templates, a form of chemical "fingerprinting". We searched the atmosphere of $51 \mathrm{Peg} b$ for molecular features arising from the expected major carbon- and oxygen-bearing gases at the observed wavelengths, namely water $\left(\mathrm{H}_{2} \mathrm{O}\right)$, carbon dioxide $\left(\mathrm{CO}_{2}\right)$, and methane $\left(\mathrm{CH}_{4}\right)$, using a grid of model atmospheres in a similar manner to Birkby et al. (2013) and de Kok et al. (2013). Spectral features from carbon monoxide (CO) are not expected in the observed $3.2 \mu \mathrm{m}$ region.

\subsection{Models}

The molecular templates are parameterized by a grid of atmospheric temperature-pressure $(T-P)$ profiles and trace gas abundances (i.e., volume mixing ratios; VMR). To generate the model spectra, we employed the same radiative transfer code of de Kok et al. (2013), performing line-by-line calculations, including $\mathrm{H}_{2}-\mathrm{H}_{2}$ collision-induced absorption along with absorption from the trace gases, which is assumed to follow a Voigt line shape. We used line data from HITEMP

\footnotetext{
11 http://www.eso.org/sci/facilities/paranal/instruments/crires/doc/VLTMAN-ESO-14500-3486_v93.pdf
} 

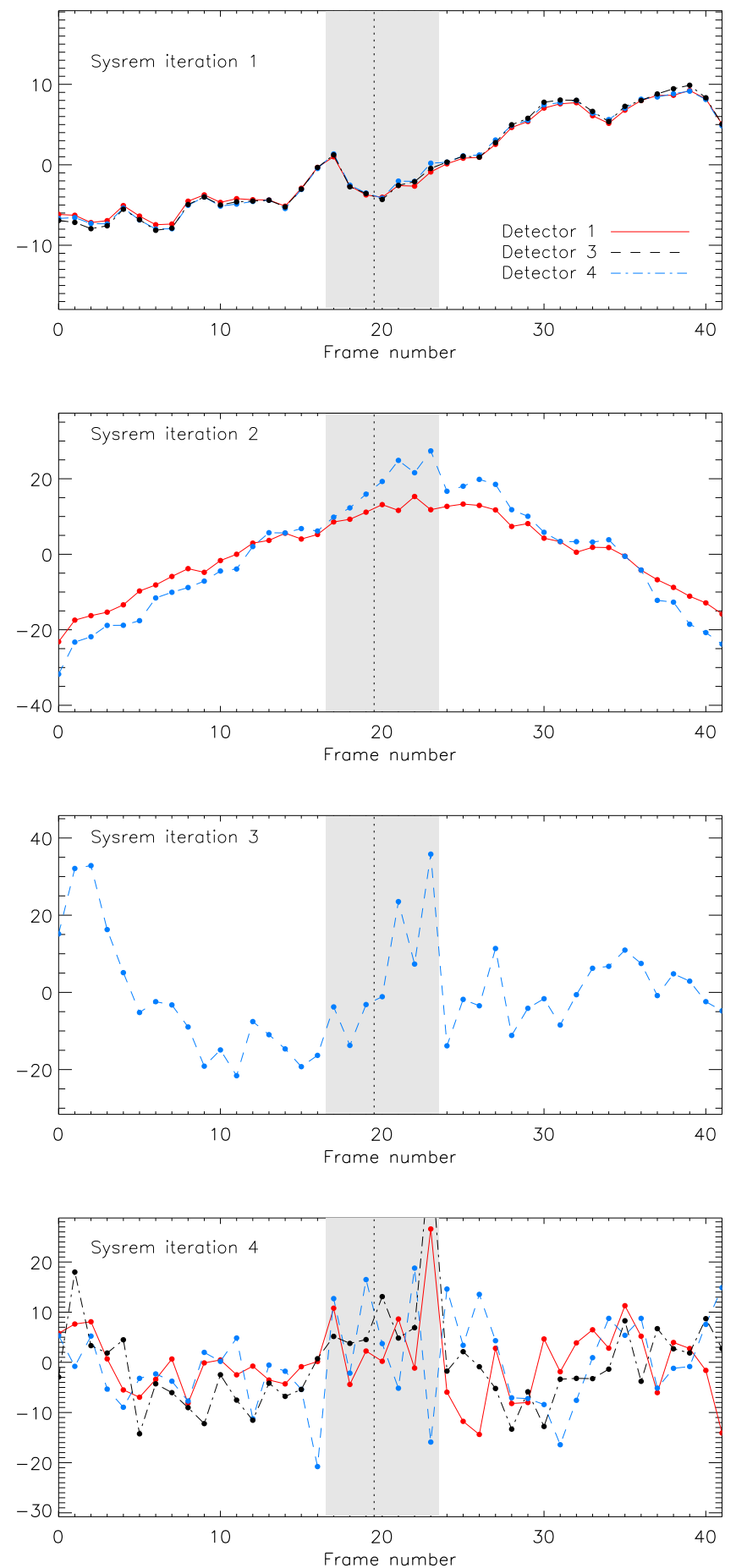

Figure 4. The trend identified and removed from each column by SYSREM for each detector. Although it is not used in our final data analysis, the fourth SYSREM iteration for all detectors is shown in the bottom panel. Its purpose here is to highlight the overall relative flatness of the removed trend, except in the gray vertical regions which bound spectra that are later excluded from the analysis of the planetary signal (see Section 3.4). These spectra occurred during a period of poor and unsettled seeing (see Figure 5). The vertical dashed line marks when the exposure time was increased from 20 to $30 \mathrm{~s}$.

2010 (Rothman et al. 2010) to create the $\mathrm{H}_{2} \mathrm{O}$ and $\mathrm{CO}_{2}$ models, and HITRAN 2008 (Rothman et al. 2009) for $\mathrm{CH}_{4}$. The model atmospheres are clear (i.e., cloud-free) with uniformly mixed gases. The $T-P$ structure follows a relatively simple profile. Deep in the atmosphere, at pressures $p_{1}$ and higher, we assume

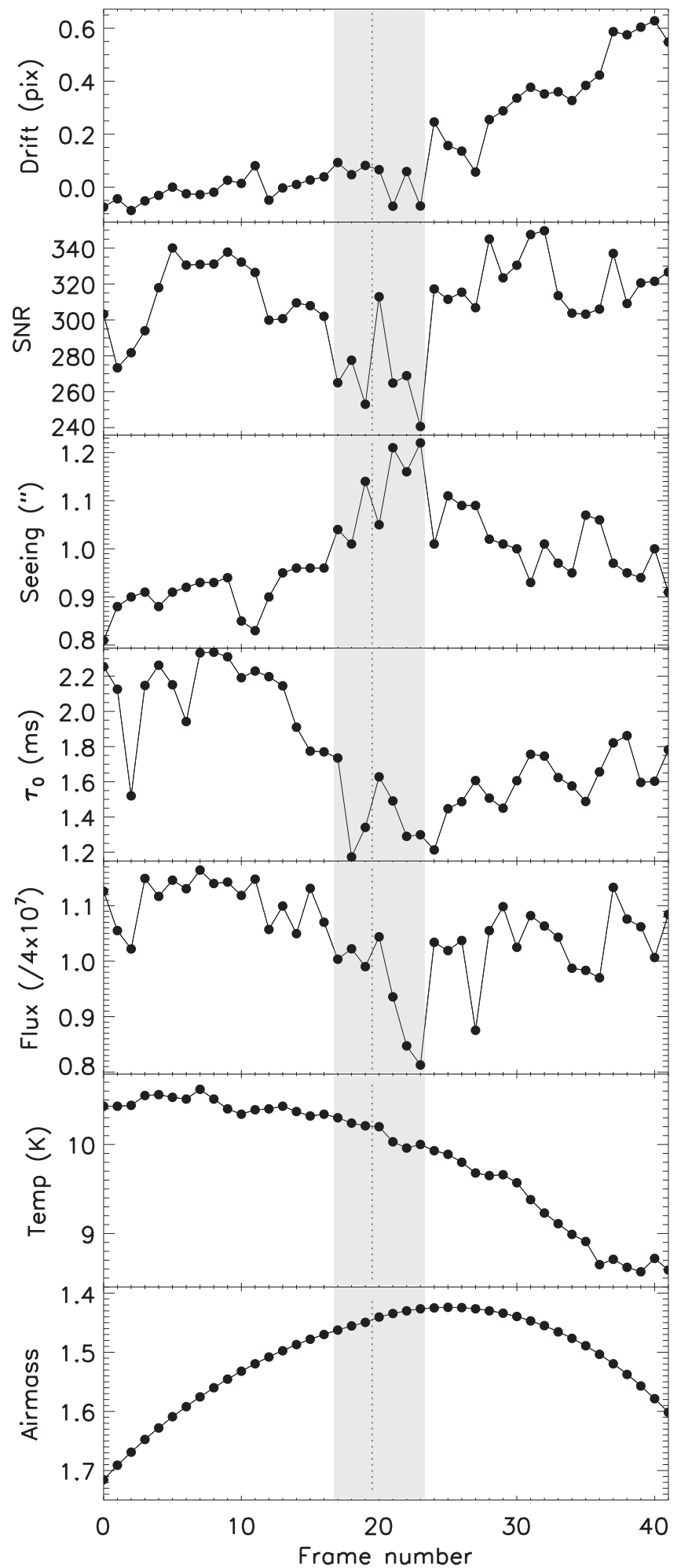

Figure 5. Possible physical causes of the trends removed by SYSREM. The gray regions and dashed vertical lines are the same as for Figure 4. Note the sudden drop in $\mathrm{S} / \mathrm{N}$ as the seeing begins to deteriorate. It recovers as the exposure time is increased but continues to degrade as the seeing worsens, only recovering when the seeing stabilizes. This trend is coincident with a rapid change in the wavefront coherence time, $\tau_{0}$. The seeing and $\tau 0$ were acquired from the VLT Astronomical Site Monitor (VLT-ASM). The flux is the raw value recorded by the adaptive optics (AO) sensor, and the temperature is the telescope ambient temperature.

a uniform $T-P$ profile at a fixed temperature $t_{1}$. Between pressures $p_{1}$ and $p_{2}$, we assume a constant lapse rate (i.e., a constant rate of change of temperature with log pressure). At 
altitudes higher (and pressures lower) than $p_{2}$ we again assume a uniform $T-P$ profile at fixed temperature $t_{2}$. The pressure $p_{1}$ took values of $(1,0.1,0.01) \mathrm{bar}$, and $p_{2}$ was varied using values of $\left(1 \times 10^{-3}, 1 \times 10^{-4}, 1 \times 10^{-5}\right)$ bar. The basal temperatures were guided by the effective temperature of the planet assuming external heating (see Equation (1) of López-Morales 2007), a low Bond albedo $\left(A_{B}<0.5\right)$, and considering the full range of heat circulation from instantaneous reradiation to full advection. Thus, $t_{1}$ took values of $(1000,1250,1500) \mathrm{K}$, while $t_{2}$ was varied using values of $(500$, $1500) K$. Note that certain combinations result in inverted $T-P$ profiles, where the temperature increases with increasing altitude (decreasing pressure). These spectra have features in emission, rather than absorption. The gas abundance volume mixing ratios took values appropriate for hot Jupiters over our considered temperature range (e.g., Madhusudhan 2012), including $10^{-4.5}, 10^{-4}$, or $10^{-3.5}$ for water, and $10^{-7}, 10^{-5}$, or $10^{-3}$ for $\mathrm{CO}_{2}$ and $\mathrm{CH}_{4}$.

Before cross-correlating the residuals with the molecular template grid, we convolved the models to the spectral resolution of CRIRES, and subtracted their baseline level. Note that the telluric removal process in Section 2.3 has also removed the continuum information in the observed planet spectrum, such that our analysis is only sensitive to the relative, not absolute, depth of the spectral features with respect to the stellar continuum.

\subsection{Cross-correlation Analysis and Results}

The cross-correlation analysis was performed for planet radial velocities in the range $-249 \leqslant R V_{\mathrm{P}}\left(\mathrm{km} \mathrm{s}^{-1}\right) \leqslant 249$ in intervals of $1.5 \mathrm{~km} \mathrm{~s}^{-1}$, interpolating the convolved grid of molecular templates onto the Doppler-shifted wavelengths. The interval size is set by the velocity resolution of the CRIRES pixels. The cross-correlation functions (CCFs) were determined separately for each residual spectrum on each detector, and then summed equally with their corresponding CCF on the other detectors, resulting in a single summed CCF matrix of dimension $333 \times 42$. The matrix created by the best-matching template is shown in upper left panel of Figure 6, where the template is a water-only model with the following parameters: $t_{1}=1500 \mathrm{~K}, t_{2}=500 \mathrm{~K}, p_{1}=0.1$ bar, $p_{2}=1 \times 10^{-5}$ bar, and a water volume mixing ratio of $\mathrm{VMR}_{\mathrm{H} 2 \mathrm{O}}=10^{-4}$. These parameters differ to the best-matching model reported by Brogi et al. (2013) for molecular absoprtion at $2.3 \mu \mathrm{m}$; however, see Sections 3.3 and 4 for further discussion on other models in the grid that produce signals within $1 \sigma$ of this result. We refer the reader to Section 3.4 for a more detailed discussion of Figure 6.

We note that in our first attempt to perform the crosscorrelation analysis of these data, we used the ephemeris and orbital solution for $51 \mathrm{Peg} \mathrm{b}$ in Butler et al. (2006), and refrained from using the phase shift invoked by Brogi et al. (2013) to match the planet signal to the systemic velocity of the host star, even though their $\Delta_{\phi}=0.0095$ phase shift was within the $1 \sigma$ uncertainty range $\Delta \phi= \pm 0.012$ of the Butler et al. (2006) ephemeris. However, we also found that this resulted in the strongest cross-correlation signal being offset from the known systemic velocity of the host star by $-9 \pm 2 \mathrm{~km} \mathrm{~s}^{-1}$, corresponding to a phase shift of $\Delta \phi=0.011$. This is still within the uncertainty of the original Butler et al.

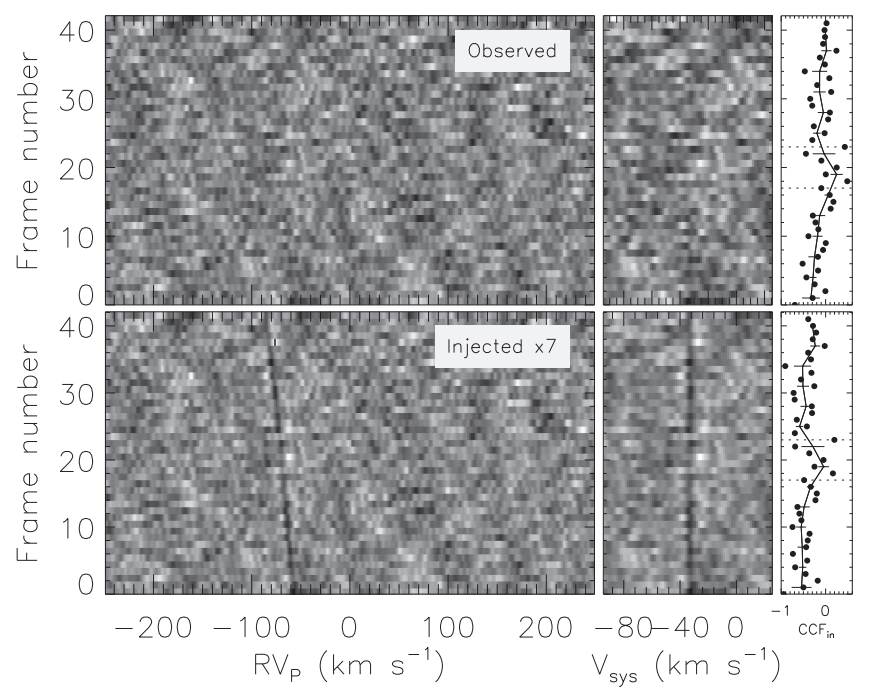

Figure 6. Cross-correlation functions for each spectrum using the bestmatching model. Top-left: the summed CCFs for each residual spectrum after cross-correlating the best-matching $\mathrm{H}_{2} \mathrm{O}$ template with the observed data. Bottom-left: same as top-left, but with a model spectrum injected at $7 \times$ its nominal value. Note the dark diagonal blueshifting trail of the injected planet signal. Top-middle: as in top left panel but aligned into the rest frame of the planet. The trail is located at the known systemic velocity of the 51 Peg star system $\left(V_{\text {sys }}=-33.25 \mathrm{~km} \mathrm{~s}^{-1}\right)$. Bottom-middle: same as topmiddle, but with the model injected. The top-right and bottom-right panels show the strength of the CCFs for a 3 pixel column bin centered on $V_{\text {sys }}=-33 \mathrm{~km} \mathrm{~s}^{-1}$ (i.e., containing the planet signal, $\mathrm{CCF}_{\text {in }}$ ). The solid line shows the mean of 3 -frame binning. Note how the strength of the CCFs approach zero between frames $18-24$ (corresponding to phase $\approx 0.565$ ). This occurs in both the observed spectra and in those where the model was injected prior to removing the telluric and stellar lines with SYSREM, and corresponds to the period of poor atmospheric conditions (see Figure 5). These CCFs are removed from our final analysis in Section 3.3, and are further detailed in Section 3.4 .

Table 1

Stellar Radial Velocity Measurements of 51 Peg from Multiple Observatories

\begin{tabular}{lccc}
\hline \hline $\mathrm{BJD}_{\mathrm{TDB}}$ & $\mathrm{RV}\left(\mathrm{m} \mathrm{s}^{-1}\right)$ & $\sigma_{\mathrm{RV}}\left(\mathrm{m} \mathrm{s}^{-1}\right)$ & Data set \\
\hline 2449610.532755 & -33258.0 & 9.0 & ELODIE \\
2449612.471656 & -33225.0 & 9.0 & ELODIE \\
2449655.311263 & -33272.0 & 7.0 & ELODIE \\
\hline
\end{tabular}

Note. The Lick8 RVs reported in this table include a $+13.1 \mathrm{~m} \mathrm{~s}^{-1}$ velocity offset correction to the RVs extracted from Vizier (http://vizier.cfa.harvard. edu/viz-bin/VizieR-3?-source=J/ApJS $/ 210 / 5 /$ table2), to account for the instrumental systematic reported in Fischer et al. (2014). The following additional offsets, determined from a circular orbit fit to each data set using EXOFAST, can be applied to place all of the RV measurements onto the same zero-point: $\gamma_{\text {Lick13 }}=-21.70 \mathrm{~m} \mathrm{~s}^{-1}, \gamma_{\text {Lick8 }}=-4.52 \mathrm{~m} \mathrm{~s}^{-1}, \gamma_{\text {Lick6 }}=$ $-14.64 \mathrm{~m} \mathrm{~s}^{-1}, \gamma_{\text {ELODIE }}=+33251.59 \mathrm{~m} \mathrm{~s}^{-1}, \gamma_{\text {HIRES }}=+2.24 \mathrm{~m} \mathrm{~s}^{-1}, \gamma_{\text {HARPS }}$ $=+33152.54 \mathrm{~m} \mathrm{~s}^{-1}$.

(This table is available in its entirety in machine-readable form.)

(2006) orbital solution. However, since the discovery of 51 Peg $\mathrm{b}$, the RV of its host star has been monitored sporadically throughout the decades, hence we endeavoured to measure the most up-to-date ephemeris for the planets orbital solution in the hope that this would negate the need for the phase shift in our analysis. 


\subsubsection{A Refined Orbital Solution for 51 Peg $b$}

To ensure we had its most up-to-date orbital solution, we compiled an extensive repository of literature and archival radial velocity measurements of the star 51 Peg from multiple observatories. These data are given in Table 1 and span observing dates from 1994 September 15 to 2014 July 9, resulting in $639 \mathrm{RV}$ measurements over 20 years. The table includes the discovery measurements from the ELODIE spectrograph at Observatoire Haute Provence (Mayor \& Queloz 1995) and subsequent additional monitoring. We took these RV measurements from the Naef et al. (2004) compilation. We also included the legacy data set from Lick Observatory observed with the Hamilton spectrograph, taking measurements from the self-consistent reprocessing of all the Lick spectra presented by Fischer et al. (2014). Finally, we included more recent additional monitoring from HIRES at the Keck Observatory (Howard \& Fulton 2016), and extracted RVs from observations with HARPS at the ESO-3.6 m telescope in 2013 (ESO program ID 091.C-0271, PI: Santos). The reduced HARPS spectra were obtained from the ESO Science Archive, ${ }^{12}$ and the RVs, their errors, and timestamps were obtained from the headers of the CCF data product files, using keywords DRS CCF RVC, DRS DVRMS, and DRS BJD, respectively. We were careful to note the format of the timestamps reported for all our data sets, which vary between JD, HJD, and BJD, due to different conventions being adopted over time. The timestamps in Table 1 were all converted to BJD $_{\text {TDB }}$ (Barycentric Dynamical Time).

We used EXOFAST ${ }^{13}$ (Eastman et al. 2013) to model the orbital components constrained by each data set. To find the best-fitting model to the radial velocity data, EXOFAST employs a nonlinear solver (AMOEBA), which uses a downhill simplex to explore the parameter space that minimizes the $\chi^{2}$ of the orbital solution. In order to negate any systematic underestimate of the uncertainties on the RV data, EXOFAST rescales the RV uncertainties by a constant multiplicative factor, such that the reduced $\chi^{2}$ of the best fit is unity $\left(\chi_{\nu}^{2}=1\right)$. Consequently, a poor fit would be reflected by larger uncertainties on the derived parameters. EXOFAST does not include an additive jitter term to the RV solution, as previous work with EXOFAST found no statistically significant difference between the two approaches to uncertainty scaling (e.g., Lee et al. 2011). Once the best fit is found, EXOFAST then executes a differential evolution Markov chain Monte Carlo method to obtain the uncertainties on the derived orbital elements. These algorithms are explained in detail in Eastman et al. (2013). The code requires priors for the stellar effective temperature $\left(T_{\text {eff }}=5787 \pm 233 \mathrm{~K}\right)$, metallicity $([\mathrm{Fe} / \mathrm{H}]=0.200 \pm 0.030)$, and surface gravity $\left(\log \left(g_{*}\right)=4.449 \pm 0.060\right)$, which we supplied based on Valenti \& Fischer (2005). We also gave the logarithm of the period $\left(\log _{10}(P)=\log _{10}(4.230785 \pm 0.000036)\right)$ as a prior based on Butler et al. (2006), and restricted the period range to 4-5 days.

Combining relative RV measurements from different observatories is hindered by velocity offsets in part due to instrumental systematics. We consider our repository to consist of six separate data sets: HARPS, HIRES, ELODIE, plus three Lick data sets (Lick13, Lick8, and Lick6). The numbers in the Lick data set names denote the dewar associated with each upgrade to the Hamilton spectrograph, which introduced different velocity offsets (see Fischer et al. 2014 for further

\footnotetext{
12 http://archive.eso.org/wdb/wdb/adp/phase3_spectral/query

13 http://astroutils.astronomy.ohio-state.edu/exofast/
}

information). Our first step was to model the data sets independently to determine if they supported an eccentric orbit. We ran two models, one with and one without a free variable for a long-term linear trend in the RVs. We excluded the HARPS data set in this assessment, as it has poor phase coverage, being clustered within $\phi= \pm 0.1$ of superior and inferior conjunction and thus lacking in strong constraint on the point of maximum absolute radial velocity. We ran the LucySweeney test to determine if the derived small eccentricities in our orbital solutions of the remaining data sets were significant (Lucy \& Sweeney 1971). The probability of small eccentricity values arising by chance were $>5 \%$ in all cases. Thus, we fixed the eccentricity to zero (circular orbits) in our subsequent modeling with EXOFAST, which allowed us to determine the velocity offset values for each data set. These offsets are listed in the notes of Table 1, and were subsequently used to place all the RVs onto the same zero-point velocity.

Prompted by the report of a $1.64 \mathrm{~m} \mathrm{~s}^{-1} \mathrm{yr}^{-1}$ trend in the RVs of 51 Peg by Butler et al. (2006) and scatter in the discovery RVs reported by Mayor \& Queloz (1995), we assessed the significance of long-term linear trends in our circular orbit solutions. We found that the earliest Lick RVs (Lick13), spanning 791 days, supported $\mathrm{a}-1.64_{-1.10}^{+1.17} \mathrm{~m} \mathrm{~s}^{-1} \mathrm{yr}^{-1}$, in agreement with Butler et al. (2006). However, the remaining Lick data sets, spanning 1175 days and 3354 days, respectively, returned non-significant linear trends of $-0.58_{-0.88}^{+0.84} \mathrm{~m} \mathrm{~s}^{-1} \mathrm{yr}^{-1}$ and $0.029_{-0.29}^{+0.28} \mathrm{~m} \mathrm{~s}^{-1} \mathrm{yr}^{-1}$, respectively. The ELODIE observations, including concurrent RVs with the Lick13 measurements, however, suggested a much smaller trend $\left(-0.15_{-0.40}^{+0.37} \mathrm{~m} \mathrm{~s}^{-1} \mathrm{yr}^{-1}\right)$, in better agreement with the more recent HIRES RVs $\left(-0.33_{-0.19}+0.19 \mathrm{~m} \mathrm{~s}^{-1} \mathrm{yr}^{-1}\right)$. It is possible that the ensemble RV data for 51 Peg probe the turnover point of an $\mathrm{RV}$ curve for a long-period companion. However, it is beyond the scope of this paper to search for such a companion. To obtain our final orbital solution for $51 \mathrm{Peg}$ b, we place all the RVs onto the zero-point described above, and model the system as a circular orbit, with no long-term linear trend. Our orbital solution is given in Table 2 and shown in Figure 7. The correlation between the parameters in the fit are shown in the Appendix along with their covariance values. We note that the additional RV scatter from any long-term companion does not cause prohibitively large error bars in the ephemeris for our later analysis.

For reference, we also show in the top panel of Figure 7 photometric monitoring data obtained with Hipparcos (ESA 1997) which were also extracted from the NASA Exoplanet Archive. The data were obtained between 1989 November and 1992 December. The plotted data only include measurements with a quality flag of zero, and are zoomed such that variations at the few percent level could be clearly seen. There are no transit events within the scatter of the data when folded on the orbital period of $51 \mathrm{Peg}$ b. This is entirely consistent with more recent ground- and space-based photometric monitoring of $51 \mathrm{Peg}$, which also report no transit events corresponding to Earth-size planets or larger at the orbital period of 51 Peg b (Guinan et al. 1995; Mayor et al. 1995; Henry et al. 1997; Walker et al. 2006). This places an upper limit on the orbital inclination angle, which is discussed in Section 4.1.

With the orbital solution updated, we re-ran the crosscorrelation analysis using our new ephemeris for $51 \mathrm{Peg}$ b. This resulted in the strongest cross-correlation signal appearing $-13.3 \pm 2 \mathrm{~km} \mathrm{~s}^{-1}$ from the known systemic velocity of the host star i.e., even further offset than when using the Butler et al. (2006) orbital calculation. In order to match the signal to the 
Table 2

Updated Orbital Solution and Planet Properties for $51 \mathrm{Peg}$ b

\begin{tabular}{|c|c|c|}
\hline Parameter & Units & Value \\
\hline \multicolumn{3}{|c|}{ Stellar } \\
\hline \multicolumn{3}{|c|}{ Parameters: } \\
\hline$M_{\star}$ & Mass $\left(M_{\odot}\right)$ & $1.100 \pm 0.066$ \\
\hline$R_{\star}$ & Radius $\left(R_{\odot}\right)$ & $1.020_{-0.079}^{+0.084}$ \\
\hline$L_{\star}$ & Luminosity $\left(L_{\odot}\right)$ & $1.05_{-0.24}^{+0.32}$ \\
\hline$\rho_{\star}$ & Density (cgs) & $1.46_{-0.27}^{+0.34}$ \\
\hline $\log \left(g_{\star}\right)$ & Surface gravity (cgs) & $4.452_{-0.059}^{+0.061}$ \\
\hline$T_{\text {eff }}$ & Effective temperature (K) & $5790 \pm 230$ \\
\hline$[\mathrm{Fe} / \mathrm{H}]$ & Metallicity & $0.198 \pm 0.029$ \\
\hline \multicolumn{3}{|c|}{ Planetary } \\
\hline \multicolumn{3}{|c|}{ Parameters: } \\
\hline$P$ & Period (days) & $4.2307869_{-0.0000046}^{+0.000045}$ \\
\hline$a$ & Semimajor axis (au) & $0.0528_{-0.0011}^{+0.0010}$ \\
\hline$T_{\text {eq }}{ }^{\mathrm{a}}$ & $\begin{array}{l}\text { Equilibrium Temper- } \\
\text { ature }(\mathrm{K})\end{array}$ & $1226_{-69}^{+72}$ \\
\hline$\langle F\rangle$ & $\begin{array}{l}\text { Incident flux } \\
\qquad\left(10^{9} \mathrm{erg} \mathrm{s}^{-1} \mathrm{~cm}^{-2}\right)\end{array}$ & $0.51_{-0.11}^{+0.13}$ \\
\hline \multicolumn{3}{|c|}{ RV Parameters: } \\
\hline$K_{\star}$ & RV semi-amplitude $\left(\mathrm{m} \mathrm{s}^{-1}\right)$ & $54.93 \pm 0.18$ \\
\hline$M_{\mathrm{P}} \sin i$ & Minimum mass $\left(M_{\mathrm{J}}\right)$ & $0.466 \pm 0.019$ \\
\hline$M_{\mathrm{P}} / M_{\star}$ & Mass ratio & $0.0004043_{-0.0000080}^{+0.0000085}$ \\
\hline$\gamma_{\mathrm{zp}}$ & $\begin{array}{l}\text { Residual zero-point off- } \\
\operatorname{set}^{b}\left(\mathrm{~m} \mathrm{~s}^{-1}\right)\end{array}$ & $0.032_{-0.077}^{+0.076}$ \\
\hline$T_{C}$ & $\begin{array}{l}\text { Time of conjunc- } \\
\text { tion }\left(\mathrm{BJD}_{\mathrm{TDB}}\right)\end{array}$ & $2456326.9314 \pm 0.0010$ \\
\hline$P_{T, G}$ & A priori transit probability & $0.092_{-0.013}^{+0.014}$ \\
\hline$M_{\mathrm{p}}$ & Planet mass $\left(M_{\mathrm{J}}\right)$ & $0.476_{-0.031}^{+0.032}$ \\
\hline$i$ & Inclination $\left(^{\circ}\right)$ & $70-90$ \\
\hline
\end{tabular}

Notes. The stellar RV parameters were derived with EXOFAST, based on priors for $\log (g), T_{\text {eff }},[\mathrm{Fe} / \mathrm{H}]$, and $\log _{10}(P)$ whose values are noted in the main text of Section 3.2.1. The eccentricity was fixed to $e=0$ and we did not allow for a long-term linear trend. The true planet mass and its range of inclination were derived from the CRIRES spectra as detailed in Section 4.1.

${ }^{\mathrm{a}} T_{\mathrm{eq}}$ was calculated assuming zero Bond albedo, $A_{B}=0$, and perfect redistribution of incident flux following Hansen \& Barman (2007).

${ }^{b}$ This is the residual scatter around the zero-point determined from the independent orbital fits. It is consistent with zero within the $1 \sigma$ error bars, and we note that the velocity resolution of the CRIRES pixels $\left(1.5 \mathrm{~km} \mathrm{~s}^{-1}\right)$ is insensitive to this small discrepancy. We adopt a literature systemic velocity of $-33.25 \mathrm{~km} \mathrm{~s}^{-1}$ (Brogi et al. 2013).

known systemic velocity, we must invoke a phase shift of $\Delta \phi=0.1$, or equivalently $\Delta T_{C}=0.07$, which is significantly larger than our $1 \sigma$ error on $T_{C}$. One therefore might conclude that the strongest cross-correlation signal is not associated with $51 \mathrm{Peg}$ b. However, given that we see a strong signal at almost identical offsets across multiple data sets (i.e., those reported here and at $2.3 \mu \mathrm{m}$ by Brogi et al. 2013), which targeted different wavelength regimes and different molecules, and underwent different data processing techniques, the case of association with the star remains plausible. While it is possible that additional long-period companions in the 51 Peg system could be affecting the orbit of $51 \mathrm{Peg}$ b, it seems unlikely that such a companion could induce the magnitude of shift in phase we have measured. We instead note that constraints on certain parameters in the orbital solution using solely the stellar RVs are not strong, especially for the argument of periastron $\left(\omega_{\star}\right)$. Using our orbital solution in Table 2, but allowing a small eccentricity $(e=0.001)$, which cannot be constrained by the existing stellar RV data, we find that an offset in $\omega_{\star}$ of just $6^{\circ}$ from the standard definition $\left(\omega_{\star}=90^{\circ}\right)$ aligns the strongest cross-correlation signal with the known systemic velocity of the host star. This is considerably smaller than the typical $\omega_{\star}$ uncertainties reported in the literature from stellar RV measurements. For example, using the Exoplanet Data Explorer ${ }^{14}$ (Han et al. 2014), we find that for similar, non-transiting hot Jupiter systems $(P<10$ days) with small orbital eccentricity $(e<0.1)$, the smallest reported error on $\omega_{\star}$ is $\pm 11^{\circ}$, and the rest are all larger than $36^{\circ}$. Given that such small changes in the orbital solution can result in alignment of the star and planet systemic velocities, we conclude that the most likely scenario arising from our data is that strongest cross-correlation signal is associated with the 51 Peg system, and thus of planetary nature. However, for simplicity, we adopt a phase shift of $\Delta \phi=0.1$ in the circular orbital solution, as noted above, to align the signal, rather than modify the eccentricity and $\omega_{\star}$.

We further note that recent studies have highlighted that the cross-correlation of water models from different molecular line list databases can result in a velocity offset due to mismatching lines (Brogi et al. 2016b). In the case of 51 Peg b, the velocity shift is seen with both water and CO models, where the latter molecule has a very robust line list. Consequently, we think it unlikely that water line lists are causing the velocity shift we observe in $51 \mathrm{Peg}$ b, but we highlight it as a potential issue for other future studies of exoplanet atmospheres at high spectral resolution.

We further conducted our cross-correlation analysis using other molecular templates containing signatures of $\mathrm{CH}_{4}, \mathrm{CO}_{2}$ for similar grid parameters, but we found no significant $(>3 \sigma)$ signal from these molecules that would indicate their presence at the abundances probed by our model grid in the atmosphere of $51 \mathrm{Peg} \mathrm{b}$ (see Section 4.2 for a discussion on the possible causes for these non-detections).

\subsection{Determining the Significance of the Cross-correlation Detection}

In the final CCF matrix the planet signal appears as a dark diagonal trail that, in this case, is blueshifting across the matrix. The trail is a small section of the planet RV curve and its slope determines the planet velocity. For visual purposes only, the bottom left panel of Figure 6 shows the CCF matrix after the best-matching template was injected into the observed spectra, before the removal of telluric contamination, at $7 \times$ its nominal value at the detected velocity of the planet. When shifted into the planet's rest frame velocity, the trail becomes vertically aligned, as shown in the middle panels of Figure 6. The size of the shift to vertically align the signal in each CCF is related to the RV semi-amplitude of the planet $\left(K_{\mathrm{P}}\right)$. Although Brogi et al. (2013) reported a tentative value of $K_{\mathrm{P}}=134.1 \pm 1.8 \mathrm{~km} \mathrm{~s}^{-1}$ for 51 Peg b, we opted to search for the planet signal over a wide range of $K_{\mathrm{P}}$ values to act as a blind search that would independently confirm this value. We therefore aligned CCFs for a range of $K_{\mathrm{P}}$ values, from $20 \leqslant K_{\mathrm{P}}\left(\mathrm{km} \mathrm{s}^{-1}\right) \leqslant 180$ in steps of $1 \mathrm{~km} \mathrm{~s}^{-1}$. To determine when the trail was vertically aligned, i.e., in the planet rest frame, thus yielding the value of $K_{\mathrm{P}}$, we performed a Welch $T$-test on each aligned CCF matrix. This statistic compared the distribution of a 3 pixel wide sliding column of "in-trail" CCFs values in the aligned matrix to the distribution of those outside it ("out-of-trail"), and determined

${ }^{14}$ http://exoplanets.org 


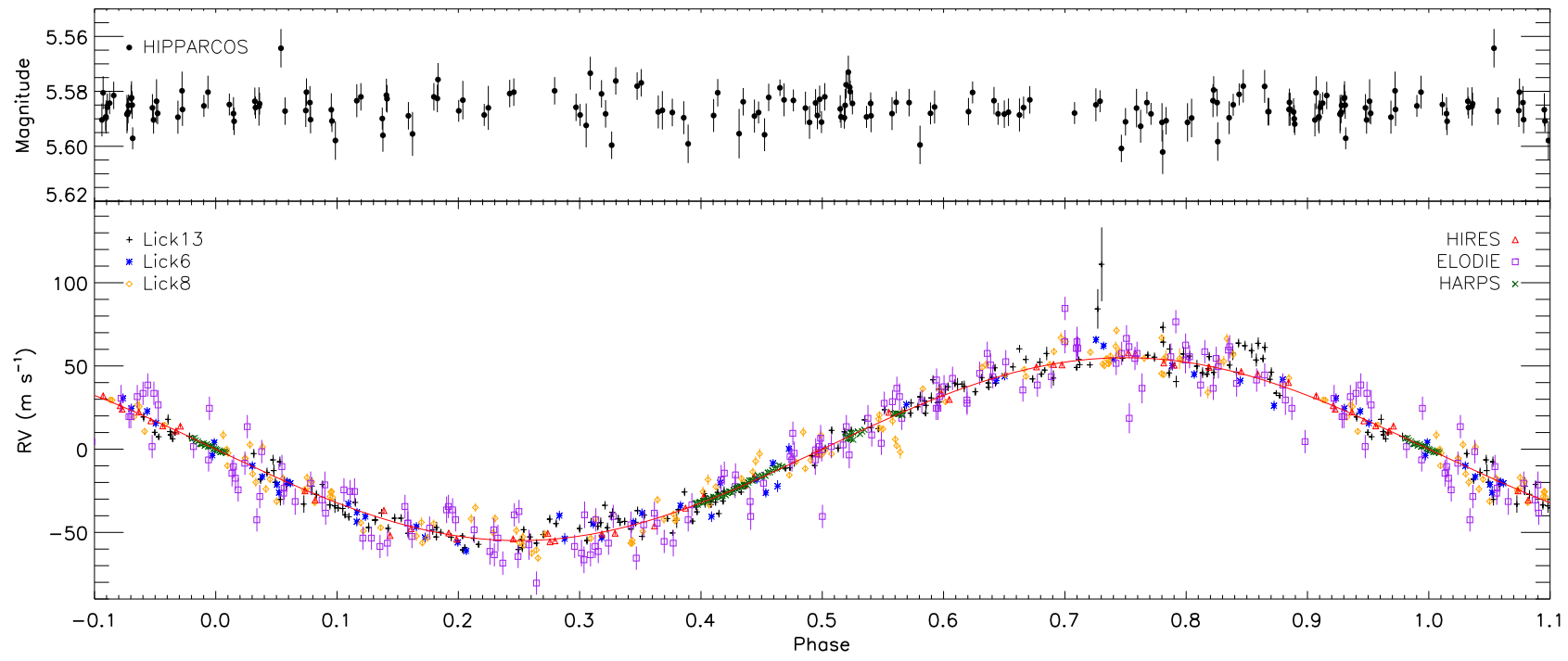

Figure 7. Top: photometric monitoring of 51 Peg from Hipparcos, phase-folded on the orbital period of 51 Peg b. The $x$-axis is plotted such that transit would occur at phase $=0$, respectively, if present in the light curve. Bottom: radial velocity curve for 51 Peg with our updated orbital solution (red solid line). The reduced $\chi^{2}$ of the best-fitting model displayed here was 6.9, thus EXOFAST rescaled all of the RV uncertainties by a multiplicative factor of 2.6 to achieve $\chi_{\nu}^{2}=1$ and counteract any underestimate of the RV uncertainties. The errors on the derived parameters in Table 2 reflect these increased RV uncertainties. The error bars in this plot have not been rescaled from the original literature values given in Table 1.

the probability that they were drawn from the same parent distribution. The sliding of the column allows for the location of $V_{\text {sys }}$, as well as $K_{\mathrm{P}}$. These probabilities were converted into $\sigma$ values, using the erf IDL function, and are displayed as a matrix in Figure 8, where $V_{\text {sys }}$ denotes the systemic velocity of the central column of the in-trail data. The most discrepant inand out-of-trail distributions deviated by $5.6 \sigma$ and corresponded to $V_{\text {sys }}=-33 \pm 2 \mathrm{~km} \mathrm{~s}^{-1}$, which is coincident with the known systemic velocity of the host star, and at $K_{\mathrm{P}}=133_{-3.5}^{+4.3} \mathrm{~km} \mathrm{~s}^{-1}$, as marked by the black cross in Figure 8 . The error bars are the $1 \sigma$ marginalized uncertainties, although it is clear they are correlated based on the ellipsoidal shape of the $1 \sigma$ error contour shown in white in Figure 8. This is expected, because as we approach the correct $K_{\mathrm{P}}$ for the planet when aligning the CCFs, the detection strength will increase. If the observations had occurred before superior conjunction, the contour would be slanted in the opposite direction. Combining multiple nights of similar data, but spanning different phase ranges, would significantly constrain the $1 \sigma$ contour (see e.g., Brogi et al. 2012). Following Brogi et al. (2014), in Figure 8 we also explore negative orbital velocities for the planet. Negative velocity implies a retrograde orbit which we know is not true for $51 \mathrm{Peg}$ b. However, any residual correlated noise that could produce a false positive would interact with the model spectrum in a similar way in both positive and negative velocity space, with a false positive appearing as a mirror image of the positive signal in the $K_{\mathrm{p}}<0 \mathrm{~km} \mathrm{~s}^{-1}$ plane. The lack of significant negative velocity signals in Figure 8 therefore serves to highlight the robustness of the positive velocity detection.

A final step in our analysis was to determine which other model water templates also provided a detection within $1 \sigma$ of the peak detection significance of $5.6 \sigma$ and thus also adequately describe the observations. Non-inverted models with shallow temperature gradients (i.e., $t_{1}=1000 \mathrm{~K}$ and $t_{2}=500 \mathrm{~K}$ ) returned comparatively lower significance values between

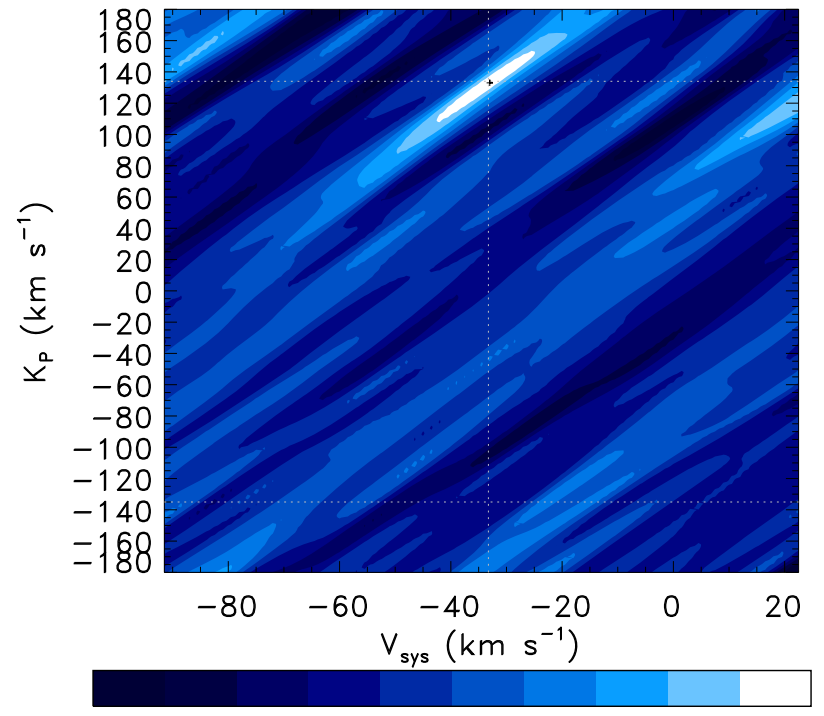

$-4.2 \sigma-3.4 \sigma-2.4 \sigma-1.4 \sigma-0.4 \sigma+0.6 \sigma+1.6 \sigma+2.6 \sigma+3.6 \sigma+4.6 \sigma+5.6 \sigma$

Figure 8. Significance values derived from the $T$-test for the best-matching template. The black plus sign marks the peak significance, $5.6 \sigma$, located at $V_{\text {sys }}=-33 \pm 2 \mathrm{~km} \mathrm{~s}^{-1}$ and $K_{\mathrm{P}}=133_{-3.5}^{+4.3} \mathrm{~km} \mathrm{~s}^{-1}$, where the errors are the maximal extent of the white $1 \sigma$ error contour. The dashed white lines mark the known systemic velocity $\left(V_{\mathrm{sys}}=-33.25 \mathrm{~km} \mathrm{~s}^{-1}\right)$, and the tentative reported value of $K_{\mathrm{P}}=134.1 \pm 1.8 \mathrm{~km} \mathrm{~s}^{-1}$ by Brogi et al. (2013). The peak significance and its $1 \sigma$ error contour are coincident with these literature values. A dashed white horizontal line at $K_{\mathrm{p}}=-134.1 \mathrm{~km} \mathrm{~s}^{-1}$ highlights that there is no matching signal in negative velocity space, acting as an additional sanity check against spurious signals.

2.4-4.5 $\sigma$. All remaining non-inverted models in the grid, which span the full range of $p_{1}, p_{2}$, and $\mathrm{VMR}_{\mathrm{H} 2 \mathrm{O}}$ that we explored, were within $1 \sigma$ of our peak detection significance. However, we found that models in our grid with a temperature inversion (i.e., $t_{2}=1500 \mathrm{~K}$ ) were all negatively correlated with 


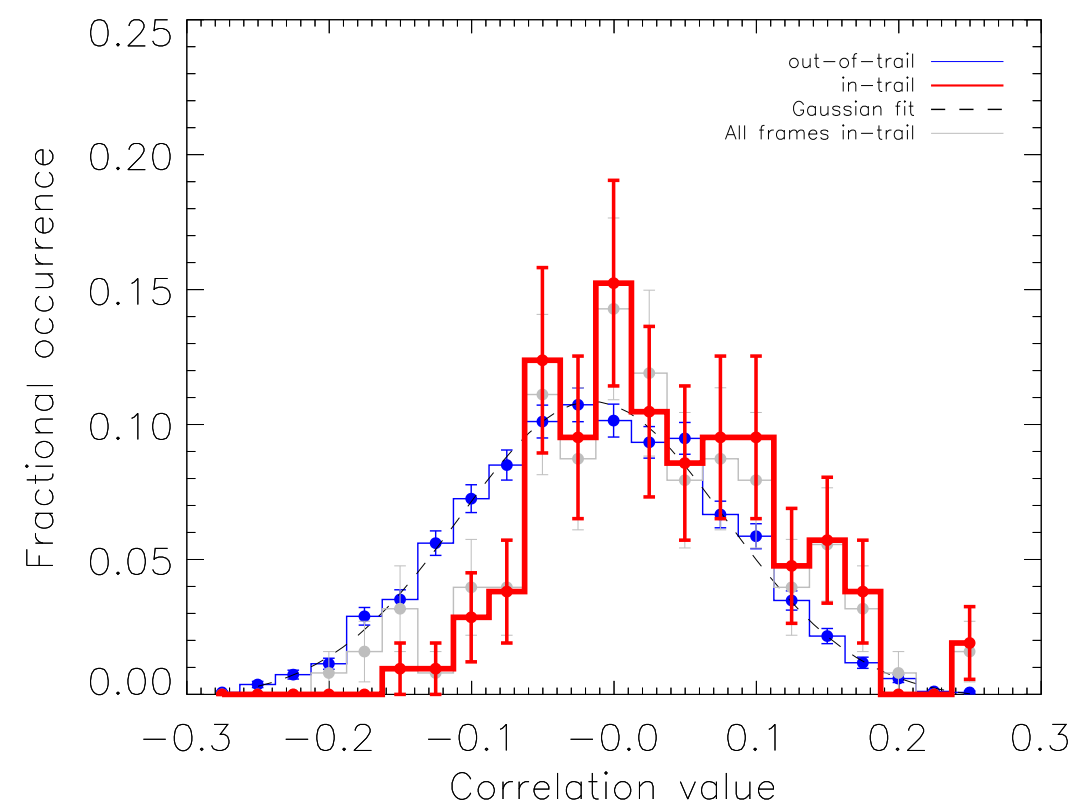

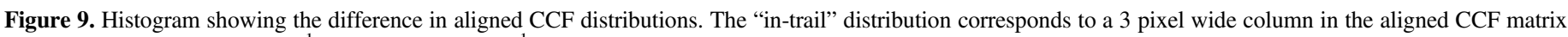

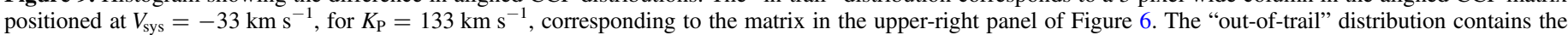

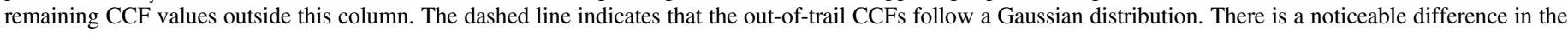

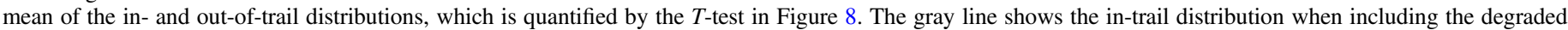

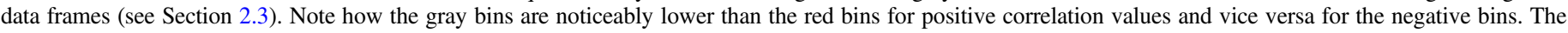

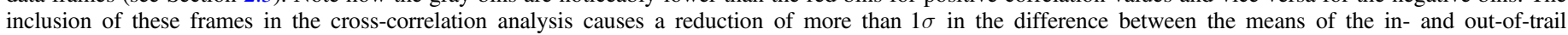
distributions. We adopt the red in-trail distribution (i.e., with the degraded frames removed) for the remainder of our analysis.

the observed planet spectrum. This means that the emission lines in the model temperature inversion spectra correlated negatively with the absorption lines in the observed planetary spectrum. Consequently, we confidently rule out the inverted models in our grid as good descriptors of the observations.

\subsection{Removal of Degraded Spectra}

A histogram of the corresponding in- and out-of-trail distributions are shown in Figure 9. Note that there are two in-trail distributions shown. The gray one includes all of the observed spectra, while the other (red) one contains only a subset as described below. The latter was used in our analysis described in Section 3.3.

We note that in the model-injected CCFs, the trail fades between frames 18-24 (see bottom-right panel of Figure 6), suggesting a fundamental degradation of the observations, and corresponds to the period of unstable seeing noted in Section 2.3. These CCFs act to scramble the distribution of the in-trail signal. Their inclusion in the $T$-test results in a $>1 \sigma$ decrease in the detection significance $(4.3 \sigma)$ compared to when they are excluded (see the gray histogram in Figure 9 and note how it shifts back toward the out-of-trail distribution). We have explored multiple reasons for why these spectra are degraded, as shown in Section 2.3. We initially attempted to weight the CCFs by the physical parameters displayed in Figure 5. However, only excessively large weightings gave an improvement, resulting in only a few CCFs dominating the final signal. Given that the injected trail also fades, we conclude that these spectra are not useful. We suspect a combination of poor atmospheric conditions and thus possible slit losses are responsible for the degradation. Indeed, Figure 5 shows that frames with the worse seeing required drift corrections on top of the temperature-induced trend, and that the flux received by the AO system is considerably lower during these times. Consequently, we chose to exclude three frames either side of the seeing spike, with the central frame being the first one with the increased exposure time. We note that this may exclude some planetary signal, thus our detection significance is conservative. The results of Section 3.3 therefore include only 35 of the observed 42 spectra.

\section{Discussion}

\subsection{Peg Ab: A Double-lined Spectroscopic Binary System}

The detection of molecular features in the atmosphere of 51 Peg b indicates that the $51 \mathrm{Peg} \mathrm{Ab}$ system is a double-lined spectroscopic binary. Our measured $K_{\mathrm{P}}$ of $51 \mathrm{Peg}$ b can be combined with $K_{\star}$ and the system mass ratio determined from precision stellar RV measurements to determine the mass of 51 Peg b, independent of its inclination, via $\frac{K_{\star}}{K_{\mathrm{p}}}=\frac{M_{\mathrm{p}}}{M_{\star}}$. Using the stellar properties given in Table 2 , the measured true mass of $51 \mathrm{Peg}$ b from our observations is $M_{\mathrm{p}}=0.476_{-0.031}^{+0.032} M_{\mathrm{J}}$, placing it firmly in the planetary mass range, and laying to rest any lingering doubts on the true nature of the first reported exoplanet orbiting a solar-like star. The planet's mass places it at the boundary between Jovian and Neptunian worlds, according to recent work by Chen \& Kipping (2016). They found data-driven evidence for a break in the power-law relationship between mass and radius for gaseous worlds at $M_{\mathrm{P}}=0.41 \pm 0.07 M_{\mathrm{J}}$. This tipping point can be physically interpreted as the mass at which any further accretion of gas into the outer layers of a Neptunian atmosphere overcomes the barrier for self-compression by gravity, leading to Jovians with 

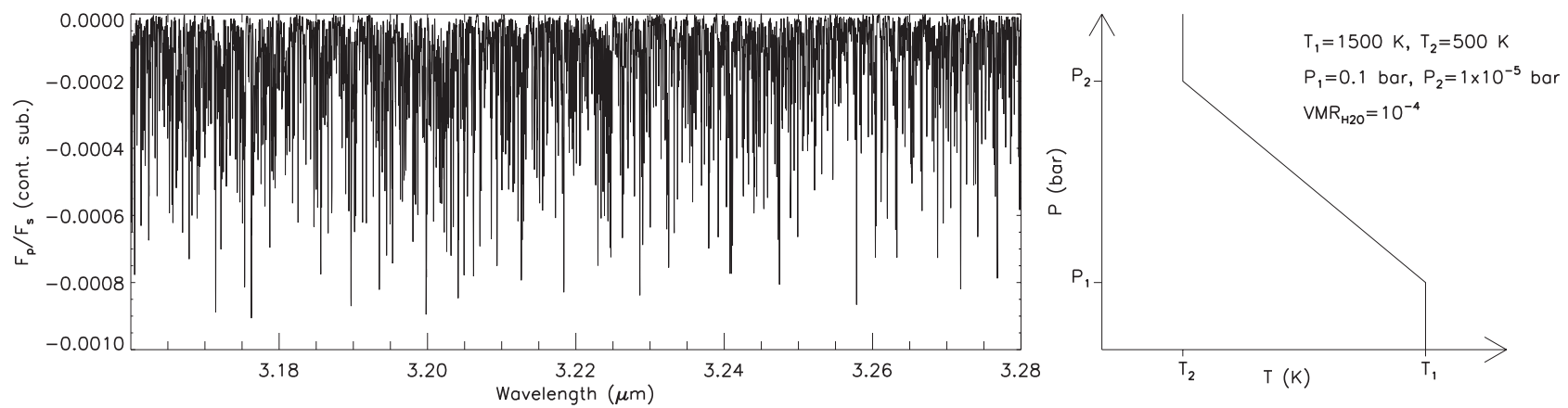

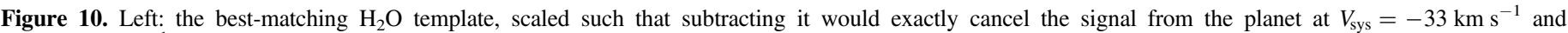

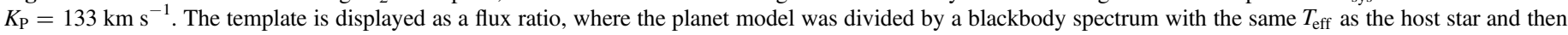

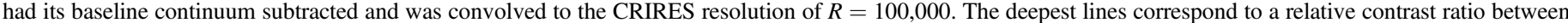

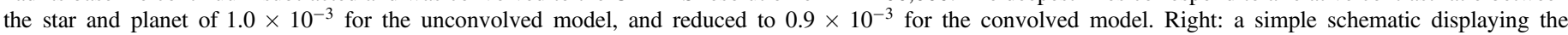
temperature-pressure profile of the best-matching template, whose properties are listed in the upper-right corner.

slightly smaller radii. Using the mass-radius relations calculated by Chen \& Kipping (2016), we predict that the radius of 51 Peg b should be between $R_{\mathrm{p}}=1.01-1.32 R_{\mathrm{J}}$, which includes a $14.6 \%$ dispersion in the mass-radius relation. We note that this is considerably smaller than the $R_{\mathrm{p}}=1.9 R_{\mathrm{J}}$ suggested by recent attempts to detect the reflected light from 51 Peg b at optical wavelengths (Martins et al. 2015), and we discuss this further in Section 4.2.2.

Now that we have measured $K_{\mathrm{P}}$ for $51 \mathrm{Peg}$ b, we can also solve for its orbital inclination using Kepler's third law, such that $P K_{\mathrm{P}} / 2 \pi a=K_{\mathrm{P}} / V_{\mathrm{P}}=\sin (i)=0.977_{0.032}^{0.038}\left(\right.$ assuming $K_{\star} \ll K_{\mathrm{P}}$, where $V_{\mathrm{P}}$ is the orbital velocity of the planet). This yields an inclination of $i=78^{\circ}$, but the $1 \sigma$ uncertainties permit values within the range $70<i\left(^{\circ}\right)<90$. The geometric probability of 51 Peg b transiting its host star is $P$ (transit) $=R_{\star} / a=9 \%$; however, the lack of transits in photometric monitoring of 51 Peg $b$ (see Figure 7) place an upper limit on the inclination angle at $i<82^{\circ} .2$, as described by Brogi et al. (2013). This is also consistent with estimates of the inclination of the stellar rotation axis at $70_{-30}^{\circ+11}$ (Simpson et al. 2010), and suggests spin-orbit alignment.

\subsubsection{Improving Ephemerides for Non-transiting Planets}

Orbital solutions for non-transiting planets are significantly more uncertain than for transiting planets, such that the epoch of periastron of non-transiting hot Jupiters can be uncertain to the order of half a day, as is the case for $51 \mathrm{Peg} b$, compared to e.g., $\pm 1.3 \mathrm{~s}$ for the transiting hot Jupiter HD $189733 \mathrm{~b}$ (Agol et al. 2010).

Although we stress that coincidental alignment of the planet and stellar systemic velocities is a strong indicator that the detected molecular absorption signal originates from the planet, in the case reported here we have multiple data sets, targeting uniquely different molecules at different wavelengths, that show molecular absorption at a consistently offset $V_{\text {sys }}$, thus providing another means to assign the signal to the planet 51 Peg b.

In fact, all of the non-transiting planets detected directly with high-resolution spectroscopy $(\tau$ Boo b, 51 Peg b, and HD 179949; Brogi et al. 2012, 2013, 2014, respectively) have required small $<1 \sigma$ phase offsets to the orbital solution from stellar RVs to place the signal at exactly the systemic velocity. Conversely, those that transited (HD 209458 b and HD 189773 b; Snellen et al. 2010; Birkby et al. 2013; de Kok et al. 2013) have not needed phase offsets, arguably due to their transits enabling far better defined ephemerides.

This leads us to the conclusion that combining precision stellar RVs with a comprehensive set of high spectral resolution planetary RVs, in a simultaneous manner, should provide strict constraints on all of the orbital elements of the star-planet system. For example, increasing $5^{\circ}$ in the argument of periastron introduces a change in the $51 \mathrm{Peg}$ stellar RVs of only a few $\mathrm{m} \mathrm{s}^{-1}$, but changes the planet RVs by several $\mathrm{km} \mathrm{s}^{-1}$. The latter is considerably easier to measure. Such precise constraints on the orbital elements allow a detailed investigation into the architecture of exoplanetary systems, as well as a more precise analysis of their circularization timescales and the tidal effects that may affect them. However, there remains an argument for continued precision $\mathrm{RV}$ monitoring of the host stars for non-transiting planets. Not only is this useful for finding additional companions, but the initial detection of the Doppler-shifting planetary signal with high-resolution spectroscopy, and thus the planet's atmospheric characterization, would be greatly aided by tightly constrained ephemerides.

\subsection{Atmospheric Properties of the Planet}

The best-matching model to the planet spectra from our grid contained molecular features from water only, with a volume mixing ratio of $\mathrm{VMR}_{\mathrm{H}_{2} \mathrm{O}}=10^{-4}$, and a $T-P$ profile that steadily reduced in temperature as altitude increased, from $t_{1}=1500 \mathrm{~K}$ at $p_{1}=0.1 \mathrm{bar}$, to $t_{2}=500 \mathrm{~K}$ at $p_{2}=1 \times 10^{-5}$ bar. This model, convolved to the $R=100,000$ resolution of CRIRES, is shown in Figure 10, along with a simple schematic of its $T-P$ structure. However, it is important to note that a number of other models give detection strengths within $1 \sigma$ of the best-matching model at velocities within the $1 \sigma$ uncertainties on $K_{\mathrm{P}}$ and $V_{\text {sys. }}$. This includes models that span the full pressure range and abundances tested by our grid. The only models in the grid we can rule out are those that include a temperature inversion within our probed pressure range, and those with $T_{1} \leqslant 1000 \mathrm{~K}$. Inversion layers, in which the bestmatching templates have emission lines, are yet to be identified with this method of atmospheric characterization (Schwarz et al. 2015). 


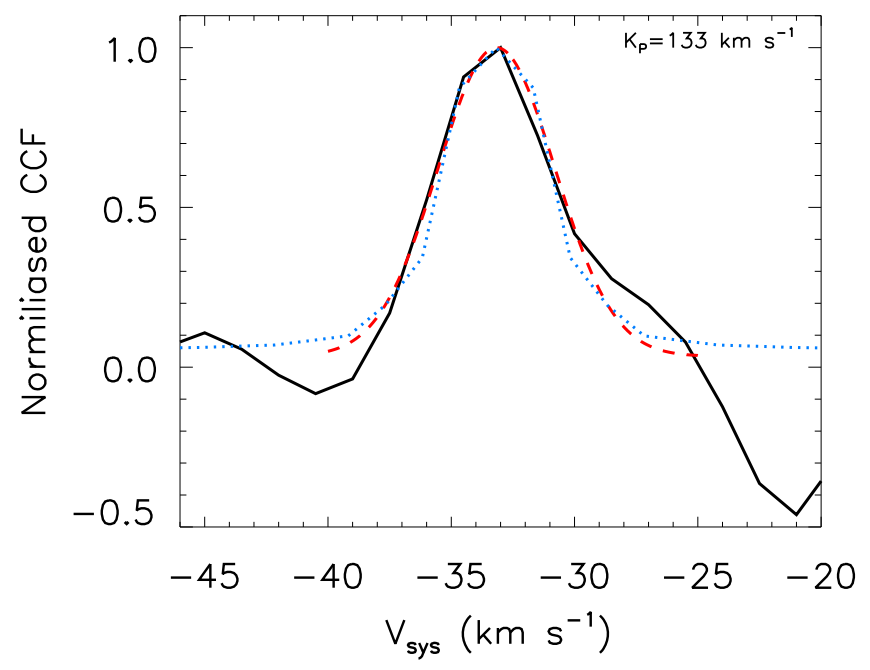

Figure 11. Section of the summed and normalized CCF for $51 \mathrm{Peg}$ b (solid black line), assuming $K_{\mathrm{P}}=133 \mathrm{~km} \mathrm{~s}^{-1}$. The dashed red line is the best-fitting Gaussian to the CCF centered at $V_{\text {sys }}=-33 \mathrm{~km} \mathrm{~s}^{-1}$. The Gaussian has a FWHM $=5.6 \mathrm{~km} \mathrm{~s}^{-1}$. The dotted blue line is the cross-correlation function of the best-matching template with itself after broadening the template to match a spectral resolution of $R=100,000$.

The deepest water lines in the best-matching template have a relative depth of $1.0 \times 10^{-3}$. This reduces to $0.9 \times 10^{-3}$ when convolved to the resolution of CRIRES. These contrast ratios were determined by scaling and injecting the negative of the best-matching template into the observed spectra before the telluric removal process, at the detected planet velocity parameters, until the peak detection significance was reduced to zero. The best-matching model shown in Figure 10 required a scaling factor of 3.0 times its nominal value to completely cancel the planet signal. The detection of $51 \mathrm{Peg} b$ via groundbased, high-resolution spectroscopy highlights the method's effectiveness in detecting faint companions among the glare of their much brighter host stars, routinely reaching contrast ratios between $10^{-4}$ and $10^{-3}$ at angular separations of $1-3 \mathrm{~ms}$.

No significant detections $(>3 \sigma)$ were seen when crosscorrelating with models containing carbon dioxide or methane. While it is likely that the abundances of these molecules are too low for these data to constrain (see e.g., Madhusudhan 2012; Moses et al. 2013 for typical abundances of hot Jupiter atmospheres as a function of temperature and $\mathrm{C} / \mathrm{O}$ ratio), we do not rule out that incorrect line positions in the line list used to create the models are responsible for their non-detection. Hot methane and other line lists are known to have inaccuracies (Hargreaves et al. 2015), particularly at high resolution (Hoeijmakers et al. 2015).

\subsubsection{Confirmation of Molecular Absorption at $2.3 \mu \mathrm{m}$}

Brogi et al. (2013) tentatively reported molecular absorption from carbon monoxide and water in the atmosphere of $51 \mathrm{Peg}$, using observations with CRIRES/VLT at $2.3 \mu \mathrm{m}$ in the same month as the observations presented here. However, Brogi et al. (2013) found that only two of their three nights of observations revealed the planet signal, with the spectral features disappearing when they should have been most visible, closest to superior conjunction when the largest fraction of dayside hemisphere is facing toward Earth. They ruled out a secondary eclipse as the cause of this as the orbital eccentricity would need to be at least $e=0.13$, which is strongly excluded by our analysis of the 51 Peg stellar RVs. Brogi et al. (2013) also investigated other causes, including several sources of instrumental instability. However, none of these could fully account for the loss of the signal. Consequently, Brogi et al. (2013) only made a tentative report of the features. Here, we have detected the orbital motion of $51 \mathrm{Peg} b$ independently, and find that our derived values for the mass, $K_{\mathrm{P}}$, and inclination are fully consistent with those reported by Brogi et al. (2013) $\left(M_{\mathrm{P}}=0.46 \pm 0.02 M_{\mathrm{J}}, K_{\mathrm{P}}=134.1 \pm 1.8 \mathrm{~km} \mathrm{~s}^{-1}\right.$, $\left.V_{\text {sys }}=-33.25 \mathrm{~km} \mathrm{~s}^{-1}\right)$. We argue that, while we used the same instrument to make our observations, our use of a different instrument set-up targeting longer wavelengths and a different molecule, alongside a different technique to remove the contaminating telluric lines, make it most likely that we have detected the same astrophysical (i.e., planetary) source, rather than both suffering from systematics at almost identical $V_{\text {sys }}$ and $K_{\mathrm{P}}$. We therefore conclude that we have affirmed the Brogi et al. (2013) detection of carbon monoxide in the atmosphere of 51 Peg b.

Following this argument, we compare our best-matching model atmospheric parameters at $3.2 \mu \mathrm{m}$ with those reported by Brogi et al. (2013) at $2.3 \mu \mathrm{m}$. The best-matching atmospheric model at $2.3 \mu \mathrm{m}$ included $\mathrm{VMR}_{C O}=10^{-4}$ and $\mathrm{VMR}_{\mathrm{H}_{2} \mathrm{O}}=3 \times 10^{-4}$, for a $T-P$ profile that is $1500 \mathrm{~K}$ at 1 bar, decreasing without inversion to $500 \mathrm{~K}$ at $10^{-5}$ bar. This is fully consistent with the $3.2 \mu \mathrm{m}$ best-matching model within the, albeit large, $1 \sigma$ uncertainties. A strong discrepancy would have highlighted that either clouds were adding to the continuum at the short wavelengths, or that the continuum probed by each wavelength region was significantly different. Such discrepancies can be ameliorated by the inclusion of wide-wavelength high-resolution observations spanning the full continuum (de Kok et al. 2014), or by the combination of external information on the true continuum level from lowresolution secondary eclipses or phase curves.

The observations at $2.3 \mu \mathrm{m}$ were taken on opposite sides of the orbit. Therefore, the slanted error contours from each night (similar to that seen in Figure 8) cross diagonally, thus narrowing the $1 \sigma$ error contour on $K_{\mathrm{P}}$ and hence reducing the $1 \sigma$ uncertainties on the planetary mass. Combining the comparatively small set of $3.2 \mu \mathrm{m}$ observations with those at $2.3 \mu \mathrm{m}$ does not improve the error bars on the orbital properties from Brogi et al. (2013), thus we refrain from including this detailed analysis in this paper. We instead highlight that comparative data sets taken at optimal wavelengths for simultaneously detecting all of the other major carbon- and oxygen-bearing molecules $\left(\mathrm{H}_{2} \mathrm{O}, \mathrm{CH}_{4}\right.$, and $\left.\mathrm{CO}_{2}\right)$ could result in more precise constraints on the relative abundance, expected to be within one order of magnitude (de Kok et al. 2014).

\subsubsection{Other Atmospheric Observations of 51 Peg $b$}

Martins et al. (2015) reported a tentative $(3 \sigma)$ detection of reflected light from 51 Peg b using high-resolution $(R=115,000)$ spectra observed with HARPS/ESO-3.6 in 2013, implying a planet-to-star flux ratio on the order of $F_{\mathrm{p}} / F_{\mathrm{s}}=10^{-4}$ at optical wavelengths. They calculated a geometric albedo of $A_{g}=0.5$ under the assumption of a highly inflated planet at $R_{\mathrm{P}}=1.9 R_{\mathrm{J}}$, while smaller planetary radii (e.g., $R_{\mathrm{P}}=1.2 R_{\mathrm{J}}$ ) would require very high albedos $\left(A_{g}>1\right)$, inconsistent with a Lambertian sphere (i.e., isotropic reflection in all directions) implying a strongly backscattering atmosphere. The CCF derived by Martins et al. (2015) for 51 Peg b was also significantly broadened to a 
FWHM $=22.6 \pm 3.6 \mathrm{~km} \mathrm{~s}^{-1}$, which at face value implies rapid rotation of the planet's atmosphere at the altitudes (pressures) probed by the optical spectra. Assuming that $51 \mathrm{Peg} \mathrm{b}$ is tidally locked to its host star, the expected rotation speed of the planet is $V_{\text {rot }}=2 \pi R_{\mathrm{P}} / P=1.2-2.3 \mathrm{~km} \mathrm{~s}^{-1}$ for $R_{\mathrm{P}}=1-1.9 R_{\mathrm{J}}$. This is close to or below the resolution of the instrument profile for both HARPS and CRIRES. We show the CCF from the CRIRES observations at the detected planet velocity $\left(K_{\mathrm{P}}=133 \mathrm{~km} \mathrm{~s}^{-1}\right)$ in Figure 11. The CRIRES CCF matches the cross-correlation of the best-matching template cross-correlated with a version of itself, after being broadened to match the $R=100,000$ resolution of CRIRES. Using the measured FWHM $=5.6 \mathrm{~km} \mathrm{~s}^{-1}$ of the CRIRES CCF, combined with the lower limit on the orbital inclination of the system and assuming the spin axes are aligned, we derive an upper limit to the rotational broadening of $51 \mathrm{Peg} b$ of $5.8 \mathrm{~km} \mathrm{~s}^{-1} \quad\left(P_{\text {rot }}>0.9\right.$ days $)$. This result applies to the atmospheric rotation at the altitude and pressure probed by our infrared observations. Rather than invoke dynamical arguments e.g., wind sheer, or an exo-ring system (Santos et al. 2015) for the discrepancy with the HARPS optical measurement of the FWHM, we instead highlight that Martins et al. (2015) urged caution regarding their FWHM measurement, stressing that injected planet spectra and the host star spectrum both resulted in overbroadened CCFs in their data analysis, possibly due to nonGaussian noise in the data, indicating that the parameters of their CCFs were strongly affected by the noise for signals at the $3 \sigma$ level.

We also note that the orbital solution used by Martins et al. (2015) assumed a fixed, zero eccentricity, but did not explore the systemic velocity parameter space, which may have led to a stronger signal being detected at the offset $V_{\text {sys }}$ we found in Section 3.2.1. If the optical data can be analyzed such that they do not induce the excess broadening seen in Martins et al. (2015), we can compare the detected planet $V_{\text {sys }}$ at optical and infrared wavelengths. If the reflected light signal is not offset in $V_{\text {sys }}$, while the infrared remains discrepant, then assuming it is astrophysical it potentially indicates an offset hot spot in the non-transiting planet's highly irradiated atmosphere, akin to that seen in the transiting hot Jupiters HD $189733 \mathrm{~b}$ and WASP-43 b (Knutson et al. 2007; Stevenson et al. 2014, respectively), enabling a means of mapping the atmospheres of close-in non-transiting planets. This would require small errors on the measured $V_{\text {sys }}$, which can be significantly reduced by observing the planet over a range of orbital phase, especially before and after superior conjunction. To date, the best constrained $V_{\text {sys }}$ of a hot Jupiter is for $\tau$ Boo b (Brogi et al. 2012) with an uncertainty of $\pm 0.1 \mathrm{~km} \mathrm{~s}^{-1}$ (an order of magnitude better than reported here for 51 Peg b). $\tau$ Boo is of similar brightness to $51 \mathrm{Peg} b$ and required three half-nights of CRIRES observations to achieve this precision on $V_{\text {sys }}$, a factor of three times longer than our $51 \mathrm{Peg}$ b observations. Similar observing durations would be required in the optical. This suggests that mapping of non-transiting hot Jupiters could be achieved with current instrumentation on $4-8 \mathrm{~m}$ class telescopes.

New and future instruments, such as ESPRESSO/VLT, and G-CLEF/GMT, will enable more sensitive probes of the reflected light and the albedo of even smaller (non-)transiting planets, while newly commissioned, improved, and upcoming high-resolution infrared spectrographs (e.g., IGRINS, GIANO, iSHELL, CARMENES, NIRSPEC, and CRIRES+, to name a few; see Crossfield 2014 for an extensive list) will independently confirm the CRIRES infrared detections to date, and probe smaller, cooler planets.

Finally, we note that dedicated, stable, uninterrupted, and consistent observing sequences under stable atmospheric conditions best serve the analysis of exoplanet atmospheres at high spectral resolution.

\section{Conclusions}

We have presented a $5.6 \sigma$ detection of water molecules in the atmosphere of the original hot Jupiter, $51 \mathrm{Peg} b$, providing the first confirmation that the $51 \mathrm{Peg} \mathrm{Ab}$ system is a doublelined spectroscopic binary. The companion orbits with a radial velocity of $K_{\mathrm{P}}=133_{-3.5}^{+4.3} \mathrm{~km} \mathrm{~s}^{-1}$, and has a measured mass of $M_{\mathrm{p}}=0.476_{-0.031}^{+0.032} M_{\mathrm{J}}$, placing it firmly in the planetary mass regime. Photometric monitoring indicates that the planet does not transit its host star, but we determine a lower limit on the inclination to be $i>70^{\circ}$. The reported observations are sensitive to small $(1 \sigma)$ changes in the parameters of the orbit solution derived from stellar RVs. This indicates that combining a comprehensive set of planet and stellar RVs would significantly improve the orbital elements. The temperature in the planetary atmosphere decreases with increasing altitude (non-inverted) over the pressure ranges probed by these $3.2 \mu \mathrm{m}$ observations. The detection of $51 \mathrm{Peg} b$ water spectral features at $3.2 \mu \mathrm{m}$ adds weight to the tentative report of $\mathrm{CO}$ and $\mathrm{H}_{2} \mathrm{O}$ molecules detected at $2.3 \mu \mathrm{m}$ by Brogi et al. (2013) in two out of three data sets, although we still cannot explain the lack of detection in their third data set by instrumental effects alone. We detected no methane or carbon dioxide at a significant level $(>3 \sigma)$ in our observations, indicating a low abundance, or possibly inaccuracies in the line lists we used to create the model templates. Our observations provide an upper limit to the rotational velocity of this non-transiting planet of $V_{\text {rot }}<5.8 \mathrm{~km} \mathrm{~s}^{-1}$, but higher instrument resolution is required to test if the planet's rotation is tidally locked to its host star. Finally, we concluded that further optical observations of 51 Peg $b$ would enable an independent orbital solution in reflected light, and if this resulted in a significantly different planet systemic velocity from the infrared observations, that an offset hot spot in the atmosphere may be needed to explain the infrared measurements. After 21 years, the detailed nature of 51 Peg $b$ is beginning to reveal itself, yet it remains an intriguing and extreme solar system.

We would like to extend our gratitude to the dedicated staff at ESO VLT for all their assistance in acquiring these data. We thank Jason Eastman for making EXOFAST freely available and easy to use, and for some helpful conversations about RVs. We also thank David Charbonneau, Mercedes Lopez-Morales, Xavier Dumusque, Matthew Kenworthy, and Stephen Roberts for helpful discussions on spectroscopy and statistics during the analysis of these data. We thank our anonymous referee whose insightful comments helped improve this manuscript. This work is based on observations collected at the European Organisation for Astronomical Research in the Southern Hemisphere under ESO programme 186.C-0289(A). This work was performed in part under contract with the Jet Propulsion Laboratory (JPL) funded by NASA through the Sagan Fellowship Program executed by the NASA Exoplanet Science Institute. Support for this work was provided in part by NASA through Hubble Fellowship grant HST-HF2-51336 awarded by the Space Telescope Science Institute, which is operated by the 
Association of Universities for Research in Astronomy, Inc., for NASA, under contract NAS5-26555. This work was part of the research program VICI 639.043.107, which is financed by The Netherlands Organisation for Scientific Research (NWO). I.A.G.S. acknowledges funding from the European Research Council (ERC) under the European Union's Horizon 2020 research and innovation programme (grant agreement nr. 694513). This research has made use of the NASA Exoplanet Archive, which is operated by the California Institute of
Technology, under contract with the National Aeronautics and Space Administration under the Exoplanet Exploration Program. This research has made use of the Exoplanet Orbit Database and the Exoplanet Data Explorer at exoplanets.org.

\section{Appendix}

In Figures 12, 13, and 14 we show the covariance between the parameters of the stellar radial velocity fit. The covariance

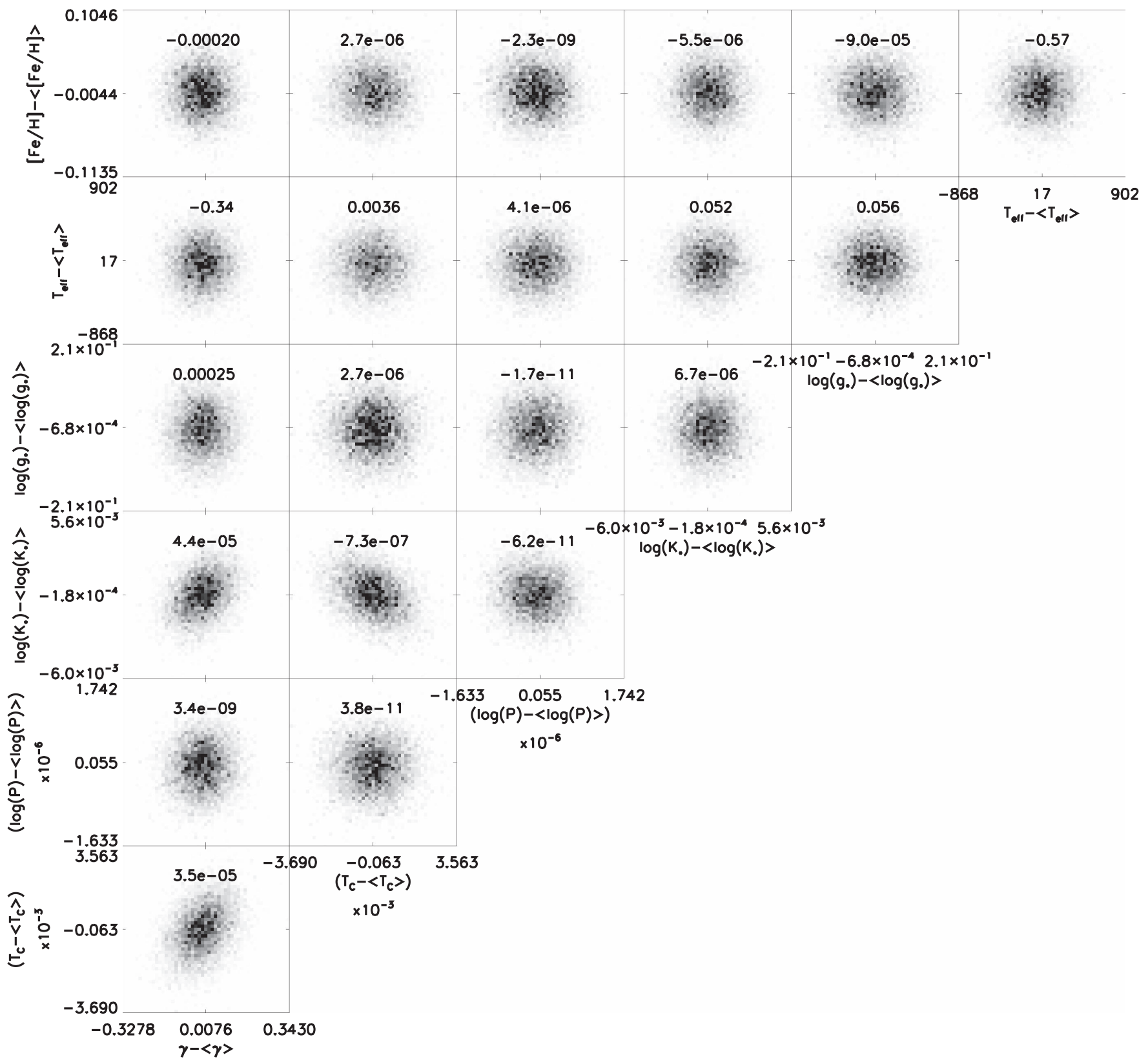

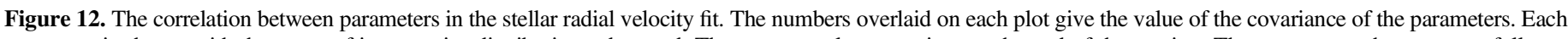

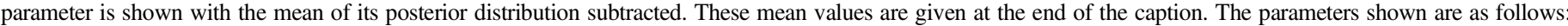

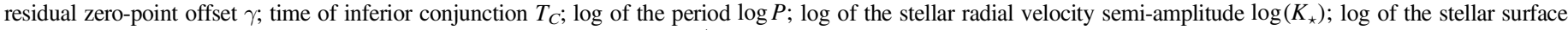

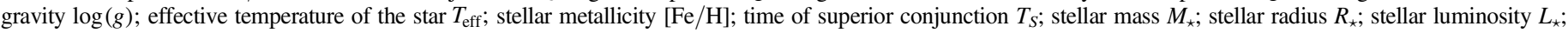

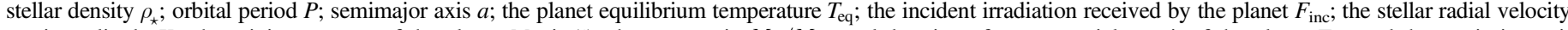

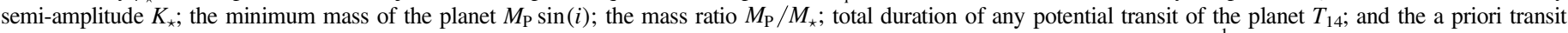

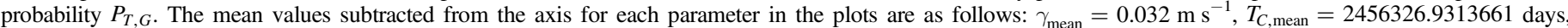

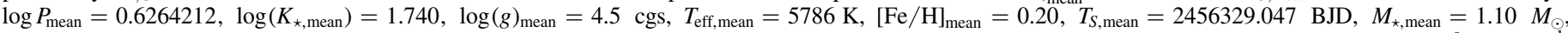

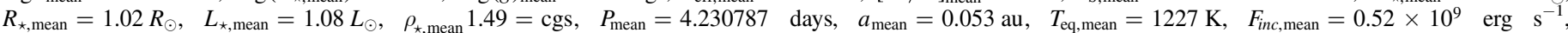
$K_{\star, \text { mean }}=54.9 \mathrm{~m} \mathrm{~s}^{-1}, M_{\mathrm{P}} \sin (i)_{\text {mean }}=0.47 M_{\mathrm{J}}, M_{\mathrm{P}} / M_{\star, \text { mean }}=0.00040, T_{14, \text { mean }}=0.12$ days, $P_{T, G, \text { mean }}=0.09$. 


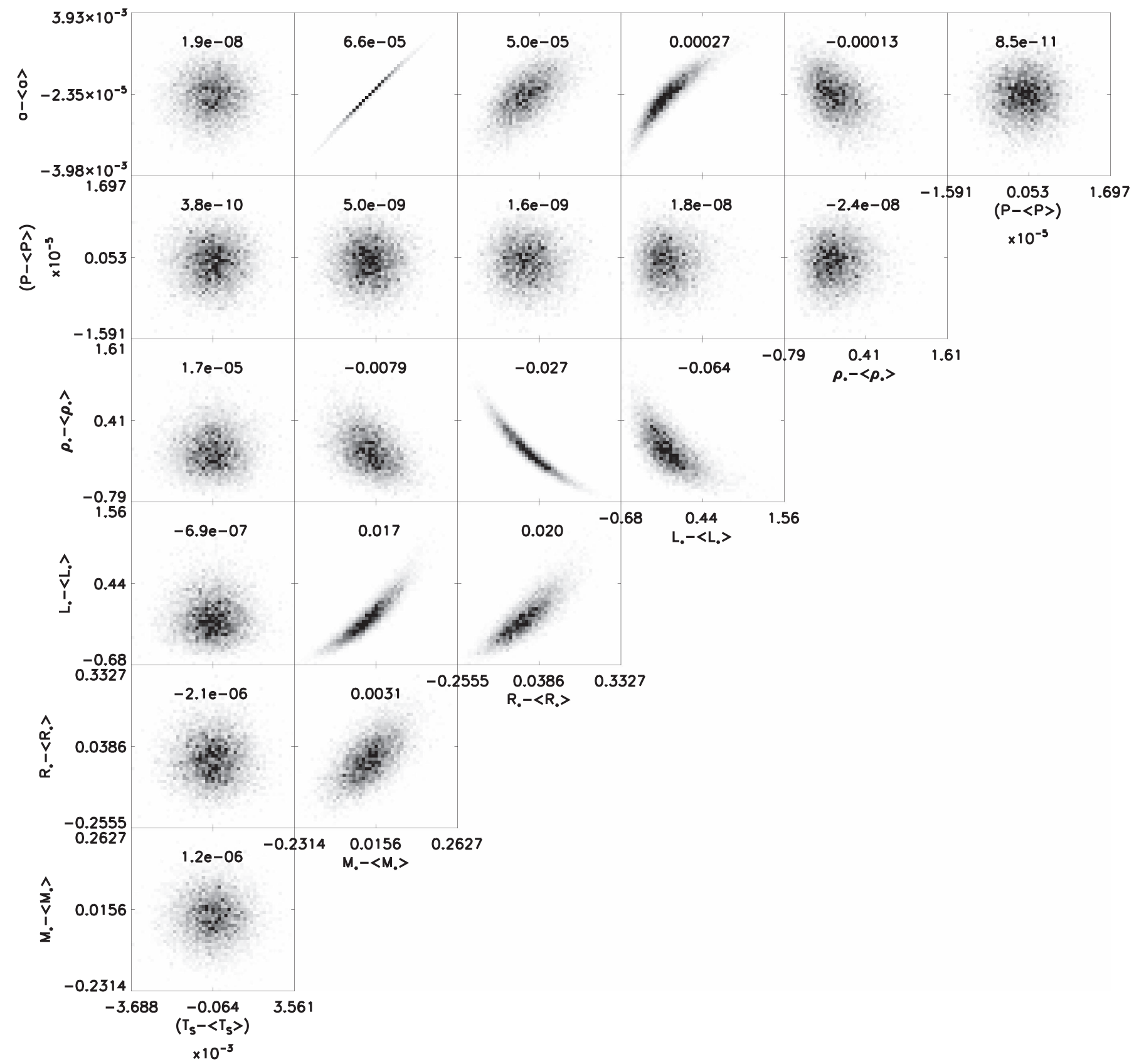

Figure 13. Same as Figure 12 but for additional parameters in the radial velocity fit.

value for each set is given on each correlation plot. Strong correlation is seen between the typically expected correlated parameters, e.g., incident flux and the equilibrium temperature of the planet, which both depend on the stellar radius, density, and surface gravity, or the times of superior and inferior conjunction. There are no strong correlations with the orbital period, nor with the residual zero-point offset. For this work, the RV semi-amplitude $K_{\star}$ and mass ratio are key parameters for determining the planet's true mass. $K_{\star}$ shows no strong correlations with other parameters, while the mass ratio shows strong correlations with the expected stellar properties, albeit with small uncertainty ranges. 


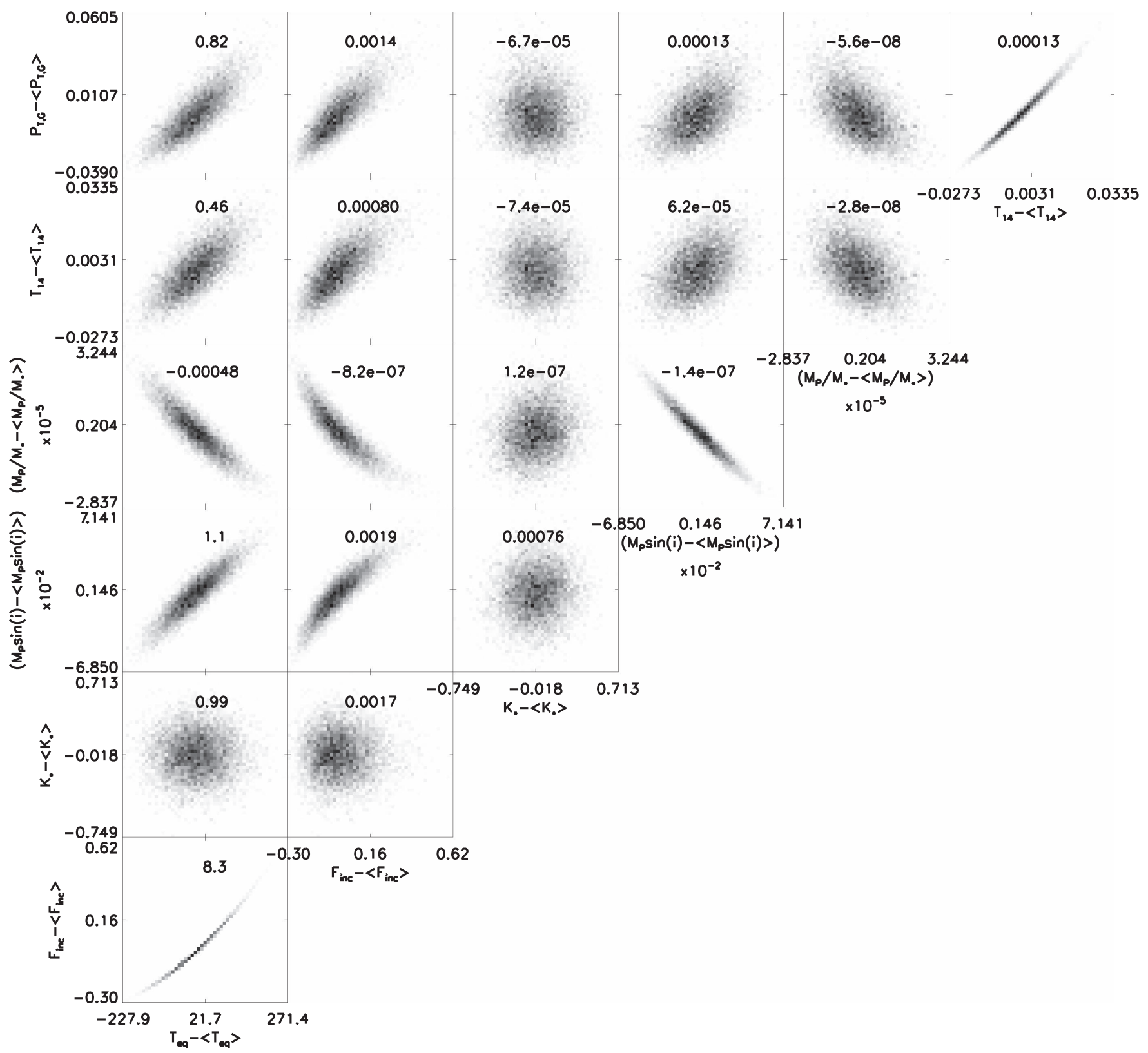

Figure 14. Same as Figures 12 and 13 but for additional parameters in the radial velocity fit.

\section{References}

Agol, E., Cowan, N. B., Knutson, H. A., et al. 2010, ApJ, 721, 1861 Arsenault, R., Alonso, J., Bonnet, H., et al. 2003, Proc. SPIE, 4839, 174 Ballard, S., Chaplin, W. J., Charbonneau, D., et al. 2014, ApJ, 790, 12 Barman, T. S., Konopacky, Q. M., Macintosh, B., \& Marois, C. 2015, ApJ, 804,61

Barnes, J. R., Barman, T. S., Prato, L., et al. 2007a, MNRAS, 382, 473

Barnes, J. R., Leigh, C. J., Jones, H. R. A., et al. 2007b, MNRAS, 379, 1097

Birkby, J. L., de Kok, R. J., Brogi, M., et al. 2013, MNRAS, 436, L35

Brogi, M., de Kok, R. J., Albrecht, S., et al. 2016a, ApJ, 817, 106

Brogi, M., de Kok, R. J., Birkby, J. L., Schwarz, H., \& Snellen, I. A. G. 2014, A\&A, 565, A124

Brogi, M., Line, M., Bean, J., Désert, J.-M., \& Schwarz, H. 2016b, arXiv:1612. 07008

Brogi, M., Snellen, I. A. G., de Kok, R. J., et al. 2012, Natur, 486, 502

Brogi, M., Snellen, I. A. G., de Kok, R. J., et al. 2013, ApJ, 767, 27

Brown, T. M. 2001, ApJ, 553, 1006

Brown, T. M., Libbrecht, K. G., \& Charbonneau, D. 2002, PASP, 114, 826
Butler, R. P., Marcy, G. W., Williams, E., Hauser, H., \& Shirts, P. 1997, ApJL, 474, L115

Butler, R. P., Wright, J. T., Marcy, G. W., et al. 2006, ApJ, 646, 505

Charbonneau, D., Brown, T. M., Latham, D. W., \& Mayor, M. 2000, ApJL, 529, L45

Charbonneau, D., Jha, S., \& Noyes, R. W. 1998, ApJL, 507, L153

Charbonneau, D., Noyes, R. W., Korzennik, S. G., et al. 1999, ApJL, 522, L145

Chen, J., \& Kipping, D. M. 2016, arXiv:1603.08614

Collier Cameron, A., Horne, K., Penny, A., \& James, D. 1999, Natur, 402, 751

Collier Cameron, A., Pollacco, D., Street, R. A., et al. 2006, MNRAS, 373, 799

Collier-Cameron, A., Horne, K., James, D., Penny, A., \& Semel, M. 2004, in IAU Symp. 202, Planetary Systems in the Universe, ed. A. Penny (Cambridge: Cambridge Univ. Press), 75

Crossfield, I. J. M. 2014, A\&A, 566, A130

Crossfield, I. J. M. 2015, PASP, 127, 941

Crossfield, I. J. M., Barman, T., \& Hansen, B. M. S. 2011, ApJ, 736, 132

Crossfield, I. J. M., Biller, B., Schlieder, J. E., et al. 2014, Natur, 505, 654 de Kok, R. J., Birkby, J., Brogi, M., et al. 2014, A\&A, 561, A150

de Kok, R. J., Brogi, M., Snellen, I. A. G., et al. 2013, A\&A, 554, A82 
Deming, D., Brown, T. M., Charbonneau, D., Harrington, J., \& Richardson, L. J. 2005, ApJ, 622, 1149

Dressing, C. D., \& Charbonneau, D. 2015, ApJ, 807, 45

Eastman, J., Gaudi, B. S., \& Agol, E. 2013, PASP, 125, 83

ESA 1997, (ed.) ESA Special Publication 1200, The HIPPARCOS and

TYCHO catalogues. Astrometric and photometric star catalogues derived from the ESA HIPPARCOS Space Astrometry Mission (Noordwijk: ESA)

Evans, T. M., Pont, F., Sing, D. K., et al. 2013, ApJL, 772, L16

Fischer, D. A., Marcy, G. W., \& Spronck, J. F. P. 2014, ApJS, 210, 5

Follert, R., Dorn, R. J., Oliva, E., et al. 2014, Proc. SPIE, 9147, 914719

Gray, D. F. 1997, Natur, 385, 795

Gray, D. F. 1998, Natur, 391, 153

Gray, D. F., \& Hatzes, A. P. 1997, ApJ, 490, 412

Guinan, E., Dukes, R., Nations, H., Buzasi, D., \& McCook, G. 1995, IAUC, 6261,2

Han, E., Wang, S. X., Wright, J. T., et al. 2014, PASP, 126, 827

Hansen, B. M. S., \& Barman, T. 2007, ApJ, 671, 861

Hargreaves, R. J., Bernath, P. F., Bailey, J., \& Dulick, M. 2015, ApJ, 813, 12

Heng, K. 2016, arXiv:1606.07218

Henry, G. W., Marcy, G. W., Butler, R. P., \& Vogt, S. S. 2000, ApJL, 529, L41

Henry, T. J., Ianna, P. A., Kirkpatrick, J. D., \& Jahreiss, H. 1997, AJ, 114, 388

Hoeijmakers, H. J., de Kok, R. J., Snellen, I. A. G., et al. 2015, A\&A, 575, A20

Howard, A. W., \& Fulton, B. J. 2016, arXiv:1606.03134

Kaeufl, H.-U., Ballester, P., Biereichel, P., et al. 2004, Proc. SPIE, 5492, 1218

Karalidi, T., Apai, D., Schneider, G., Hanson, J. R., \& Pasachoff, J. M. 2015, ApJ, 814, 65

Knutson, H. A., Charbonneau, D., Allen, L. E., et al. 2007, Natur, 447, 183

Knutson, H. A., Charbonneau, D., Cowan, N. B., et al. 2009, ApJ, 690, 822

Konopacky, Q. M., Barman, T. S., Macintosh, B. A., \& Marois, C. 2013, Sci, 339, 1398

Kostov, V., \& Apai, D. 2013, ApJ, 762, 47

Kreidberg, L., Bean, J. L., Désert, J.-M., et al. 2014, Natur, 505, 69

Lee, B. L., Ge, J., Fleming, S. W., et al. 2011, ApJ, 728, 32

Lin, D. N. C., Bodenheimer, P., \& Richardson, D. C. 1996, Natur, 380, 606

Lockwood, A. C., Johnson, J. A., Bender, C. F., et al. 2014, ApJL, 783, L29

López-Morales, M. 2007, ApJ, 660, 732

Lord, S. D. 1992, NASA Technical Memorandum,103957

Louden, T., \& Wheatley, P. J. 2015, ApJL, 814, L24

Lucy, L. B., \& Sweeney, M. A. 1971, AJ, 76, 544

Macintosh, B., Graham, J. R., Barman, T., et al. 2015, Sci, 350, 64
Madhusudhan, N. 2012, ApJ, 758, 36

Martins, J. H. C., Santos, N. C., Figueira, P., et al. 2015, A\&A, 576, A134

Mayor, M., \& Queloz, D. 1995, Natur, 378, 355

Mayor, M., Queloz, D., Marcy, G., et al. 1995, IAUC, 6251, 1

Mazeh, T., Tamuz, O., Zucker, S., et al. 2007, in ASP Conf. Ser. 366

Transiting Extrasolar Planets Workshop, ed. C. Afonso, D. Weldrake, \& Th. Henning (San Francisco, CA: ASP), 119

Moses, J. I., Madhusudhan, N., Visscher, C., \& Freedman, R. S. 2013, ApJ, 763,25

Naef, D., Mayor, M., Beuzit, J. L., et al. 2004, A\&A, 414, 351

Piskorz, D., Benneke, B., Crockett, N. R., et al. 2016, arXiv:1609.09074

Rasio, F. A., \& Ford, E. B. 1996, Sci, 274, 954

Rodler, F., \& López-Morales, M. 2014, ApJ, 781, 54

Rodler, F., Lopez-Morales, M., \& Ribas, I. 2012, ApJL, 753, L25

Rodler, F., Kürster, M., \& Barnes, J. R. 2013, MNRAS, 432, 1980

Rothman, L. S., Gordon, I. E., Barbe, A., et al. 2009, JQSRT, 110, 533

Rothman, L. S., Gordon, I. E., Barber, R. J., et al. 2010, JQSRT, 111, 2139

Santos, N. C., Martins, J. H. C., Boué, G., et al. 2015, A\&A, 583, A50

Schneider, J., Dedieu, C., Le Sidaner, P., Savalle, R., \& Zolotukhin, I. 2011, A\&A, 532, A79

Schwarz, H., Brogi, M., de Kok, R., Birkby, J., \& Snellen, I. 2015, A\&A, 576, A111

Schwarz, H., Ginski, C., de Kok, R. J., et al. 2016, A\&A, 593, A74

Simpson, E. K., Baliunas, S. L., Henry, G. W., \& Watson, C. A. 2010, MNRAS, 408, 1666

Sing, D. K., Fortney, J. J., Nikolov, N., et al. 2016, Natur, 529, 59

Snellen, I., de Kok, R., Birkby, J. L., et al. 2015, A\&A, 576, A59

Snellen, I. A. G., Brandl, B. R., de Kok, R. J., et al. 2014, Natur, 509, 63

Snellen, I. A. G., de Kok, R. J., de Mooij, E. J. W., \& Albrecht, S. 2010, Natur, 465, 1049

Snellen, I. A. G., de Kok, R. J., le Poole, R., Brogi, M., \& Birkby, J. 2013, ApJ, 764,182

Stevenson, K. B., Désert, J.-M., Line, M. R., et al. 2014, Sci, 346, 838

Tamuz, O., Mazeh, T., Zucker, S., et al. 2005, MNRAS, 356, 1466

Valenti, J. A., \& Fischer, D. A. 2005, ApJS, 159, 141

Walker, G. A. H., Matthews, J. M., Kuschnig, R., et al. 2006, in Tenth Anniversary of 51 Peg-b: Status of and Prospects for Hot Jupiter Studies, ed. L. Arnold, F. Bouchy, \& C. Moutou (Paris: Frontier), 267

Zhou, Y., Apai, D., Schneider, G. H., Marley, M. S., \& Showman, A. P. 2016, ApJ, 818, 176 